\title{
Epigenetic regulation of the lineage specificity of primary human dermal lymphatic and blood vascular endothelial cells
}

\author{
Carlotta Tacconi $^{1}$ (D) $\cdot$ Yuliang He $^{1} \cdot$ Luca Ducoli $^{1} \cdot$ Michael Detmar $^{1}$ (D)
}

Received: 10 April 2020 / Accepted: 1 September 2020 / Published online: 12 September 2020

(c) The Author(s) 2020

\begin{abstract}
Lymphatic and blood vascular endothelial cells (ECs) share several molecular and developmental features. However, these two cell types possess distinct phenotypic signatures, reflecting their different biological functions. Despite significant advances in elucidating how the specification of lymphatic and blood vascular ECs is regulated at the transcriptional level during development, the key molecular mechanisms governing their lineage identity under physiological or pathological conditions remain poorly understood. To explore the epigenomic signatures in the maintenance of EC lineage specificity, we compared the transcriptomic landscapes, histone composition (H3K4me3 and H3K27me3) and DNA methylomes of cultured matched human primary dermal lymphatic and blood vascular ECs. Our findings reveal that blood vascular lineage genes manifest a more 'repressed' histone composition in lymphatic ECs, whereas DNA methylation at promoters is less linked to the differential transcriptomes of lymphatic versus blood vascular ECs. Meta-analyses identified two transcriptional regulators, BCL6 and MEF2C, which potentially govern endothelial lineage specificity. Notably, the blood vascular endothelial lineage markers CD34, ESAM and FLT1 and the lymphatic endothelial lineage markers PROX1, PDPN and FLT4 exhibited highly differential epigenetic profiles and responded in distinct manners to epigenetic drug treatments. The perturbation of histone and DNA methylation selectively promoted the expression of blood vascular endothelial markers in lymphatic endothelial cells, but not vice versa. Overall, our study reveals that the fine regulation of lymphatic and blood vascular endothelial transcriptomes is maintained via several epigenetic mechanisms, which are crucial to the maintenance of endothelial cell identity.
\end{abstract}

Keywords Blood endothelial cells $\cdot$ Lymphatic endothelial cells $\cdot$ Cell identity $\cdot$ Epigenetics $\cdot$ DNA methylation $\cdot$ Histone modifications

\section{Introduction}

The lymphatic and the blood vasculature exert complementary functions in humans. While the blood vasculature represents a closed circulatory system essential for the delivery

Carlotta Tacconi and Yuliang He have equally contributed to this work.

Electronic supplementary material The online version of this article (https://doi.org/10.1007/s10456-020-09743-9) contains supplementary material, which is available to authorized users.

Michael Detmar

michael.detmar@pharma.ethz.ch

1 Institute of Pharmaceutical Sciences, Swiss Federal Institute of Technology, ETH Zurich, Vladimir-Prelog-Weg 3, HCI H303, 8093 Zurich, Switzerland of oxygen and nutrients, the lymphatic circulation is responsible for draining interstitial fluid from peripheral tissues, also serving as a conduit for immune cell trafficking and lipid absorption in the gut [1]. Both types of vasculature form highly branched networks that are lined by endothelial cells (ECs). Blood capillaries, which are characterized by continuous inter-endothelial junctions, are surrounded by pericytes embedded in a basement membrane. In contrast, lymphatic capillaries are thin-walled, highly permeable blind-ended vessels that lack pericyte coverage and a continuous basement membrane. These capillaries converge to form larger collecting vessels surrounded by mural cells. Collecting lymphatic vessels are furnished with intraluminal valves that enable the unidirectional transport of lymph back to the blood circulation through the lymphovenous valve junctions located around the jugular region [2]. 
Despite distinct functions of the blood vascular and the lymphatic endothelia, they share a close developmental relationship. During early mammalian embryonic development, endothelial progenitors differentiate from the mesodermal tissue to form a primitive vascular system. This ancestral vascular plexus then undergoes remodeling, resulting in endothelial sprouting (angiogenesis) and specification into arterial or venous cell fates. After the formation of arteries and veins, the first lymphatic ECs (LECs) transdifferentiate from the cardinal vein [3]. Lymphatic development occurs in a stepwise process, where a subset of ECs starts to express COUP-TFII and SOX18, sequentially inducing the expression of the prospero-related homeobox 1 transcription factor (PROX1) [4]. PROX1 is considered to be one of the "master regulators" during lymphatic development and it is the PROX1-positive cells that commit to the lymphatic lineage $[5,6]$. Upon terminal differentiation, LECs express several lineage-specific markers, including PROX1, the transmembrane glycoprotein podoplanin (PDPN) and the vascular endothelial growth factor receptor 3 (VEGFR-3 or FLT4) [7, 8]. The conservation of EC identities is crucial for specialized vasculature functions. However, ECs retain, at least experimentally to some extent, the capacity to transdifferentiate into the alternate endothelial lineage, by repression of genes that determine the pre-existing cell fate and/or by activating the opposing markers [9]. Such endothelial plasticity has also been observed under pathological conditions in vivo [10]. For instance, in inflammation and cancer, blood vessels can change phenotypes by expressing lymphatic-specific molecules such as VEGFR-3 [11, 12]. The molecular mechanisms underlying EC differentiation during development have been comprehensively studied, both in terms of marker expression and signaling pathways. However, the mechanisms maintaining EC lineage specificity during postnatal life are still not fully understood.

Activation or suppression of transcription is a highly regulated process that can be orchestrated by epigenetic modifications. Emerging epigenome studies have advanced our understanding of the crosstalk between diverse epigenetic mechanisms and the acquisition of different biological traits during tissue development [13] and under pathological conditions $[14,15]$. While recent efforts have been made to uncover the importance of epigenomic architecture for vascular quiescence and specification [16, 17], the epigenetic mechanisms in the maintenance of EC identity remain to be fully elucidated. This has thus fostered our interest in characterizing the epigenetic regulations of the lymphatic versus the blood vascular endothelial lineages. In the present study, we performed comparative analyses of the transcriptomic profiles of blood vascular ECs (BECs) and LECs and of their DNA methylomes and the landscapes of histone modifications. Our results indicate that endothelial lineagespecific markers have specific epigenetic profiles that maintain lineage identity.

\section{Results}

\section{Distinct transcriptomic patterns of lymphatic and blood vascular endothelial cells}

We and others have previously reported that BECs and LECs are characterized by distinct expression patterns of lineagespecific markers $[18,19]$. To gain a comprehensive view of their differential transcriptomes and to identify key regulatory factors, we first performed deep RNA sequencing of cultured primary human dermal BECs and LECs derived from two individual donors. The purity of isolated cells was confirmed by FACS analysis of the pan-endothelial marker PECAM1 and PDPN. Both LECs and BECs showed high positivity for PECAM1. However, only LECs exhibited a strong positivity for PDPN, while BECs were completely negative for this lymphatic marker (Fig. S1a). The independent biological replicates exhibited high pairwise correlations (Fig. S1b, c). Both cell types conserved high expression levels of the classical EC markers PECAM1 and CDH5 (Fig. S1d). Comparative transcriptomic analysis identified 2382 differentially expressed genes $(\log 2 \mathrm{FC}>1$ and FDR $<0.01)$ between BECs and LECs (Fig. 1a). In consistence with the current view of lineage-specific markers, BECs displayed high levels of CD34, ESAM and FLT1 (Fig. 1b), while LECs specifically expressed PROX1, PDPN and FLT4 (Fig. 1c). Differential expression patterns of these lineage-specific markers were further confirmed by qPCR (Fig. S1e, f). Despite recent efforts in depicting the epigenetic landscape of various EC types, very few transcriptomic and DNA methylation signatures pinpointing BEC and LEC identities have been reported [20, 21]. Interestingly, we identified a list of 42 epigenetic regulators, which have previously been described as molecules involved in chromatin remodeling, histone modifications and DNA methylation $[22,23]$ to be differentially expressed in either EC type (Fig. 1d), implicating a potential role of epigenetic modifications in the maintenance of EC lineage specificity.

\section{Endothelial lineage specificity is in part regulated at the level of histone modifications}

Post-translational modification of histone proteins can markedly affect DNA accessibility to transcriptional regulators. For instance, trimethylation of different lysine residues at the $\mathrm{N}$-terminus may induce either active or repressed chromatin composition [24]. To assess the importance of histone modifications in the maintenance of EC identity, we conducted 
a

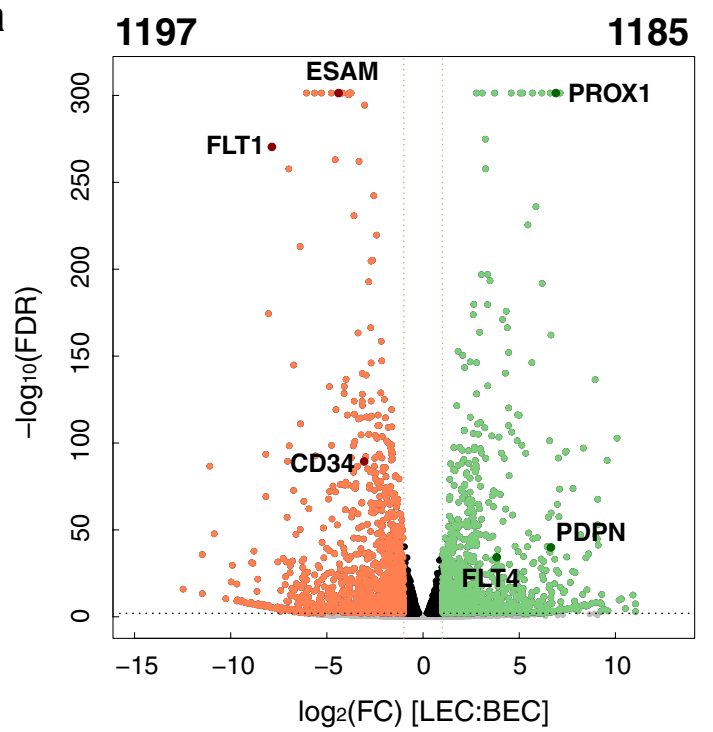

d

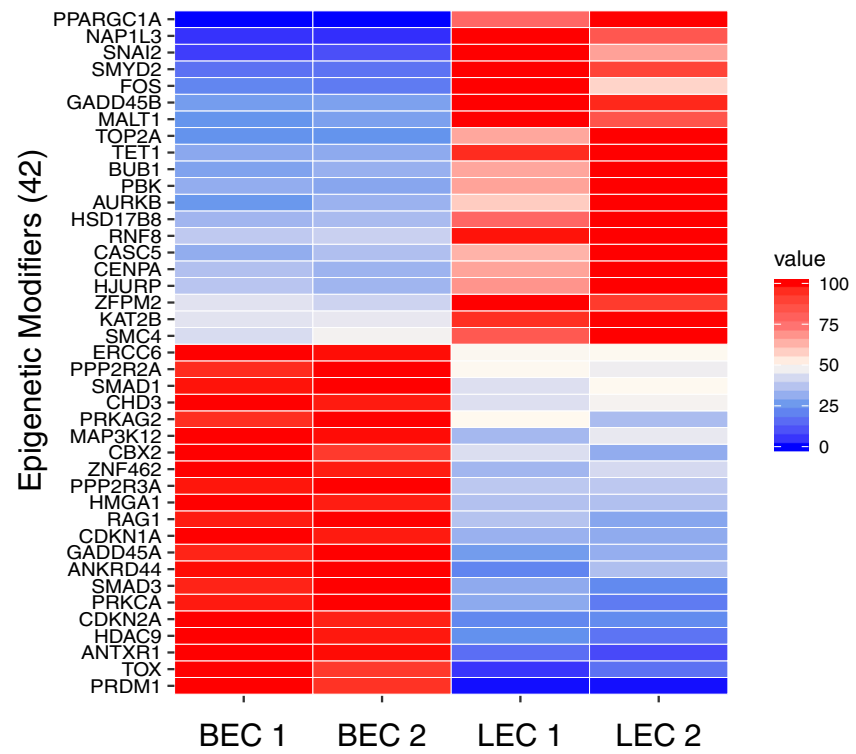

b CD34

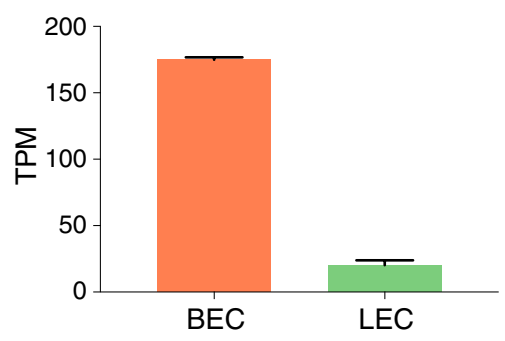

C

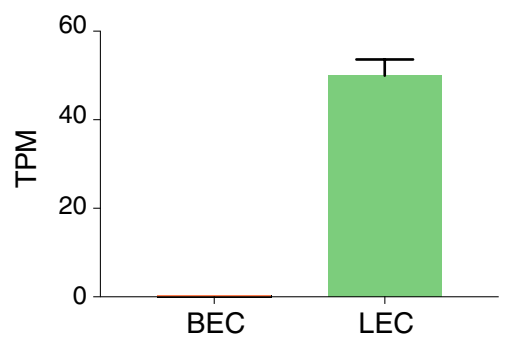

ESAM

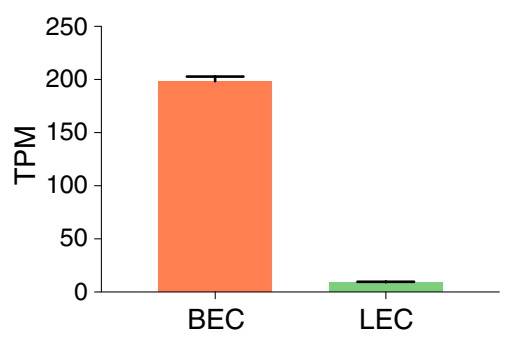

PDPN

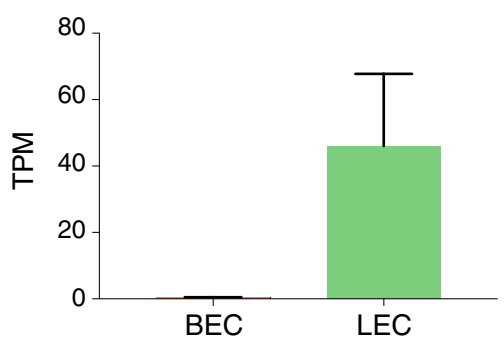

FLT1

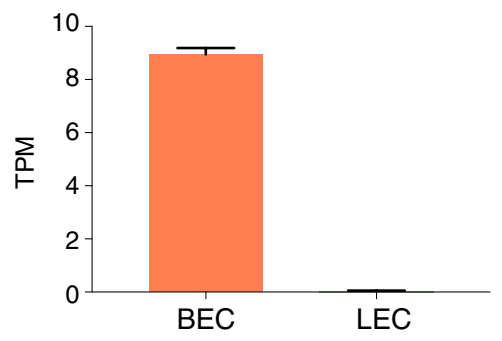

FLT4

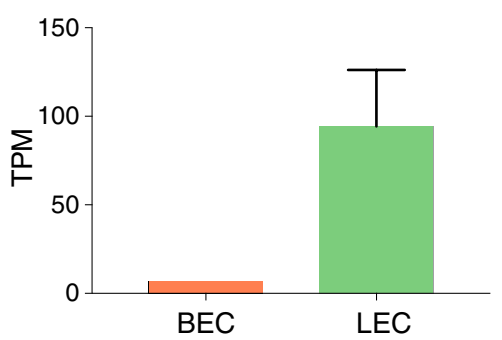

Fig. 1 BECs and LECs display lineage-specific transcriptomic landscapes. a Volcano plot showing the upregulated genes in BECs (orange) and LECs (green), defined by $\log 2$ fold-change (FC) $(>1)$ and FDR $(<0.01)$. b and $\mathbf{c}$ Expression levels (in Transcripts Per Million, TPM) of the lineage-specific BEC (CD34, ESAM and FLT1)

Chromatin Immunoprecipitation Sequencing (ChIP-Seq) profiling of the H3K4me3 and H3K27me3 histone marks, which are enriched in active or repressed chromatin regions, respectively $[25,26]$. Correlation analyses of aligned reads revealed good reproducibility between the two biological replicates (Fig. S2a, b). H3K4me3 was preferentially and LEC markers (PROX1, PDPN and FLT4). d Heatmap depicting the expression pattern of the differentially expressed epigenetic modifiers in each cell type. Values are shown as the percentage of maximum expression of the respective gene

enriched at promoter regions, while $\mathrm{H} 3 \mathrm{~K} 27 \mathrm{me} 3$ was more widely spread out and marked distal intergenic regions in a comparable fashion in both cell types (Fig. S2c). To better understand the connection between differential enrichment of histone modifications at promoter regions and the lineage-specific gene expression patterns, we graded the 
a

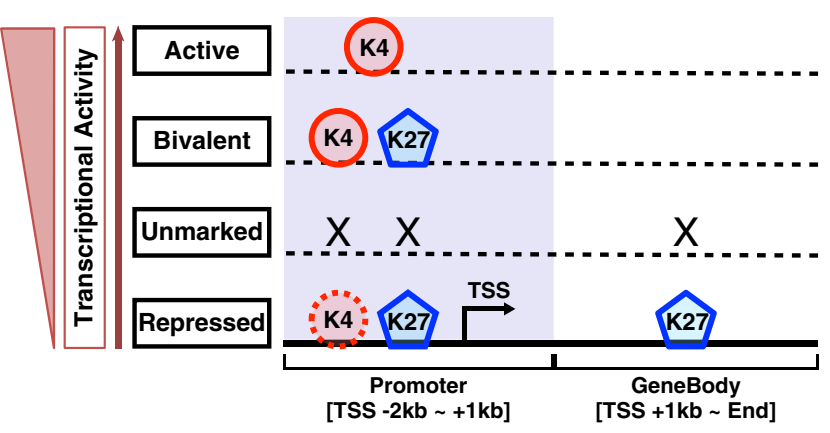

d Percentage of differentially expressed genes

$\begin{array}{lllll}0 & 25 & 50 & 75 & 100\end{array}$

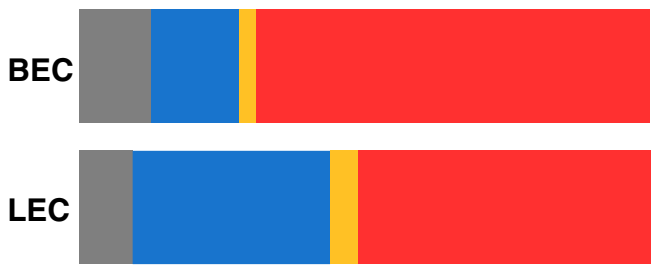

f

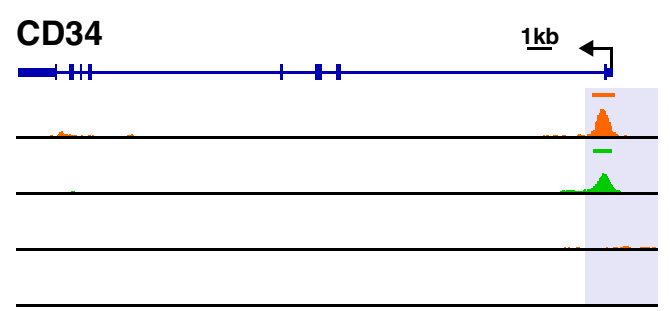

ESAM

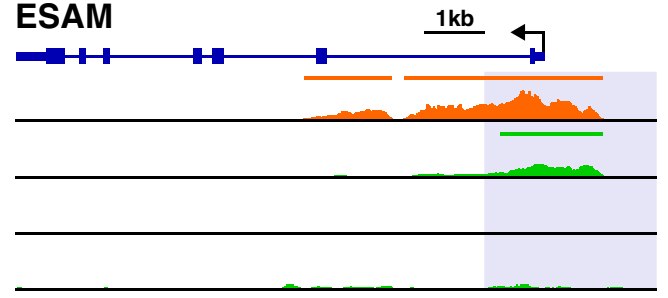

FLT1

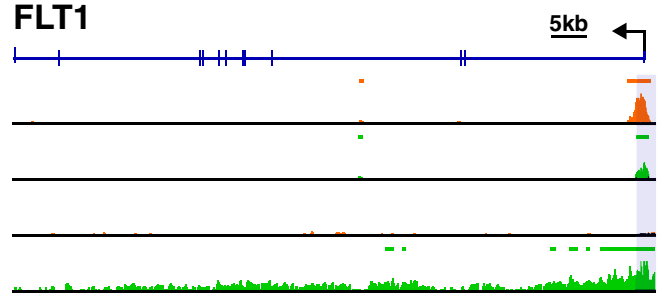

b

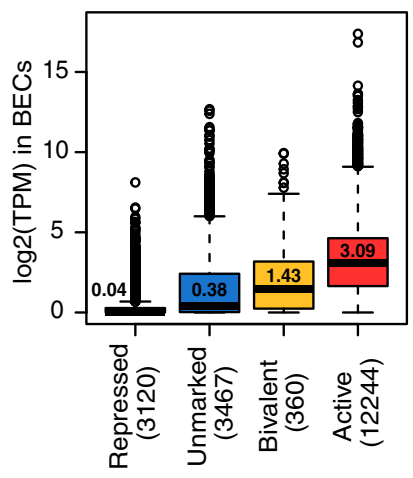

C LEC

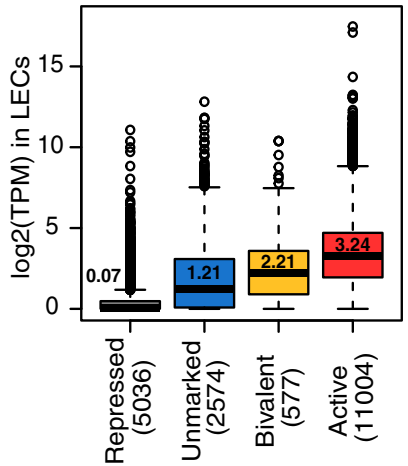

e Percentage of differentially expressed genes

$\begin{array}{lllll}0 & 25 & 50 & 75 & 100\end{array}$

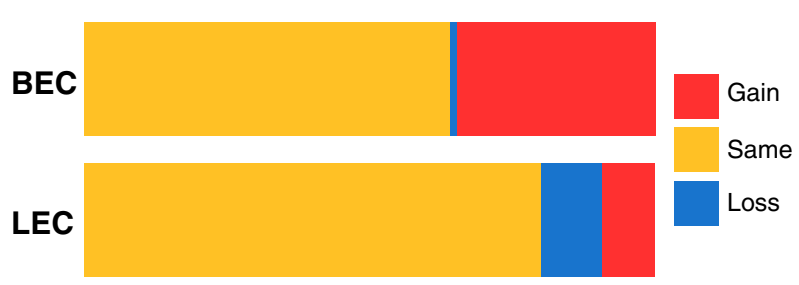

g

BEC H3K4me3

LEC H3K4me3

BEC H3K27me3

LEC H3K27me3

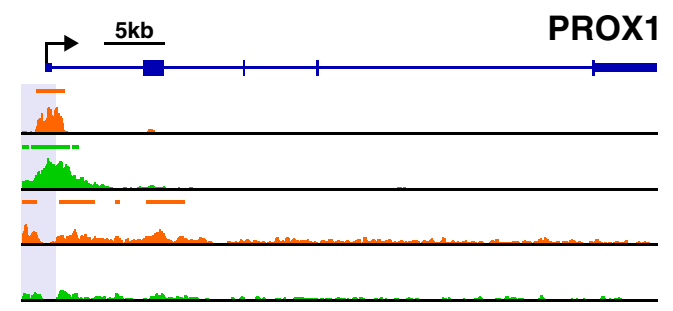

BEC H3K4me3

LEC H3K4me3

BEC H3K27me3

LEC H3K27me3

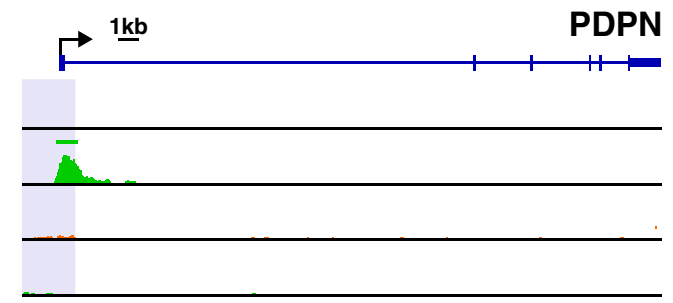

FLT4

$1 \underline{\mathbf{k b}}$

BEC H3K4me3

LEC H3K4me3

BEC H3K27me3

LEC H3K27me3 histone enrichment states into 4 levels in an incremental order of transcriptional activity (Fig. 2a). Namely, the 'repressed' state denoted by the coverage of H3K27me3 across the gene body or the spanning of H3K27me 3 over the promoters in the absence of $\mathrm{H} 3 \mathrm{~K} 4 \mathrm{me} 3$; the 'unmarked' state, where the whole transcript is devoid of any histone 
४Fig. 2 LECs and BECs present different H3K4me3 and H3K27me3 enrichment profiles. a Representation of the chromatin composition in each histone state, which was graded by their transcriptional activity. The black arrow labels the transcription start site (TSS) and the direction of the transcription. The promoter region is highlighted in blue. $\mathbf{b}$ and $\mathbf{c}$ Expressed genes were categorized into the corresponding histone state in BECs and LECs. Transcriptomic abundances were compared among different histone states and the number of genes in each group is indicated in brackets. The numbers inside the box plots denote the medians. d Overview of the histone state of genes with upregulated expression in the respective cell type as determined in Fig. 1a. e Proportion of upregulated genes manifesting a sustained or switched histone state, in contrast to the opposing cell type. Differential histone enrichment profiles of the selected BEC (f) and LEC (g) marker genes. The magnitude of the signal tracks of a given histone modification for a given marker is the same across two cell types. Orange/green bars at the top of each track indicate the called ChIPSeq peaks

marks; the 'bivalent' state with both histone modifications residing in the promoter regions; and lastly the 'active' state when the promoters are solely enriched for the H3K4me3 mark (Fig. 2a).

The expression level of genes detected in each EC type was in line with the activity of their histone enrichment states (Fig. 2b, c). We then evaluated the histone states of the genes with increased expression in the respective cell type. The majority of upregulated genes were found in an 'active' histone state (69\% and 51.2\% respectively in BECs and LECs), and both cell types showed a similar proportion of upregulated genes in the 'unmarked' or the 'bivalent' state (Fig. 2d). We next investigated if increased gene expression could be attributed to enhanced transcriptional activity of the histone states. We considered the switch of a more favorable histone composition in one cell type compared to the other as a 'gain' of transcriptional activity. Genes with the same histone state were also treated as 'gain' of activity in the presence of stronger $\mathrm{H} 3 \mathrm{~K} 4 \mathrm{me} 3$ or weaker H3K27me3 enrichments, and vice versa as a 'loss' (Fig. 2e). Surprisingly, only $9.2 \%$ of the upregulated genes in LECs resulted from a 'gain' of histone states, with another $10.2 \%$ showing a 'loss' of favorable histone modifications (Fig. 2e). In contrast, a much lower percentage (1.2\%) of upregulated genes in BECs displayed a 'loss' of histone state (Fig. 2e). Over one third $(34.7 \%)$ of the genes enriched in BECs had an enhanced transcriptional activity of their histone states, whereas they were conversely maintained at a more 'repressed' state in LECs (Fig. 2e, Supplementary Table 1). A closer look at the organization of histone marks of selected lymphatic and blood vascular EC markers revealed that all of them were in the 'active' state in the respective cell type. As for the BEC markers, both ESAM and FLT1 acquired a 'gain' of histone states in BECs, signified by stronger enrichment and broader coverage of $\mathrm{H} 3 \mathrm{~K} 4 \mathrm{me} 3$ over the transcription start site (TSS), whereas H3K27me3 expanded into the FLT1 gene body exclusively in LECs (Fig. 2f). Likewise, the lymphatic markers PROX1, PDPN and FLT4, were devoid of the H3K27me3 marks and manifested a 'gain' of histone states in LECs, whereas they adopted either 'unmarked' or 'bivalent' states in BECs (Fig. 2g). CD34 exhibited 'active' histone composition in both BECs and LECs with similar levels of $\mathrm{H} 3 \mathrm{~K} 4 \mathrm{me} 3$ enrichment at the promoter region.

Collectively, we found that the endothelial lineage markers present differential enrichment profiles of $\mathrm{H} 3 \mathrm{~K} 4 \mathrm{me} 3$ and H3K27me3 histone composition in BECs and LECs. In particular, a large number of blood vascular markers manifest a more repressive histone state in LECs, implicating a potential epigenetic mechanism in the maintenance of lymphatic lineage specificity.

\section{Promoter hypomethylation accounts to a lesser extent for the differential transcriptomes}

Besides epigenetic regulations via histone modifications, DNA methylation is also of importance in regulating gene expression and cellular plasticity [27]. In fact, cells acquire specific DNA methylation patterns during the process of differentiation [28], where heavily methylated promoter regions in principle correlate with repressed transcription [29]. To investigate the potential relevance of DNA methylation in maintaining endothelial lineage specificity, we next examined the EC methylomes using the Infinium MethylationEPIC array. We observed a strong correlation between the methylation profiles of the biological replicates (Fig. S3a, b). Differential methylation analysis identified 19,139 loci $(2.4 \%$ of probes) in BECs and 10,687 loci (1.3\% of probes) in LECs to be hypomethylated in the respective cell type (Fig. 3a). It is notable that 4917 hypomethylated loci detected in BECs and 2751 in LECs lay within enhancer regions, constituting about $26 \%$ of the differentially methylated probes (Fig. 3a, Fig. S3c, d). To integrate the DNA methylation profiles with the transcriptomic landscape, we dissected the methylation patterns of differentially expressed genes, in accordance with the genic locations from upstream promoter regions to the 3' untranslated regions (UTRs) (Fig. 3b, c). Although the upregulated genes primarily had a lowly methylated state around the promoters in the respective cell type, we did not observe a major shift in the methylation status in the opposing cell type at any genic region (Fig. 3b, c). In addition to interrogating separately the methylation levels of individual $\mathrm{CpG}$ loci, we also evaluated the variations in differentially methylated regions (DMRs) comprised of multiple consecutive $\mathrm{CpG}$ sites. Overall, there was only a modest agreement between increased expression and hypomethylation of the corresponding promoter in BECs (104 genes; Fig. 3d) and LECs (51 genes; Fig. 3e, Supplementary Table 2). Hypomethylated DMRs residing in the promoter regions were detected for CD34 and ESAM in BECs, and for PROX1 in LECs, in line with their increased expression in 


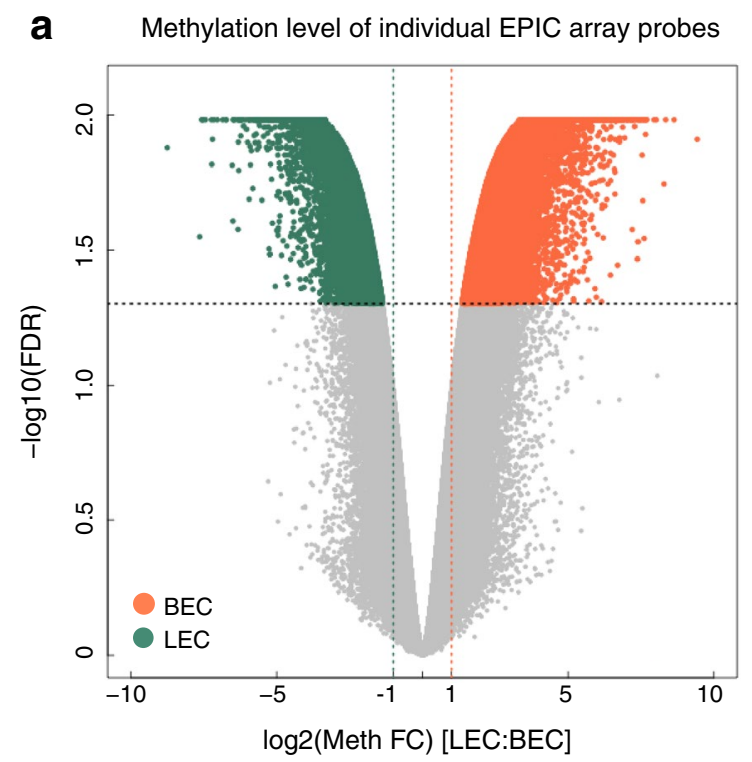

b Methylation level by genic region (BEC genes)
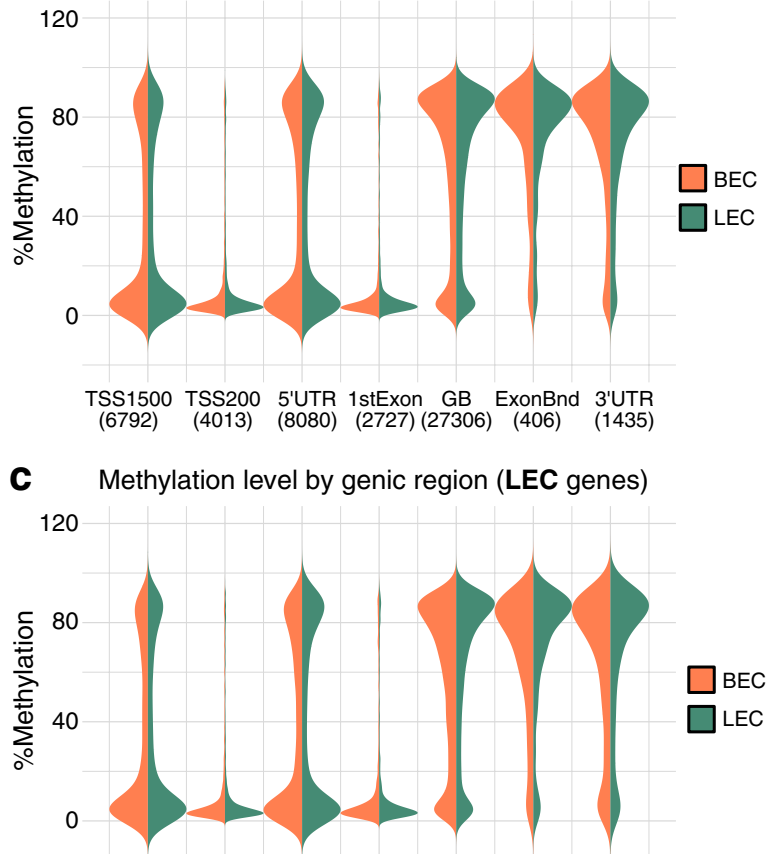

d

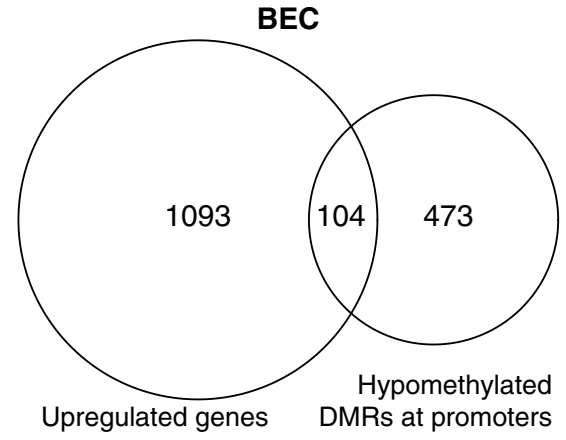

e

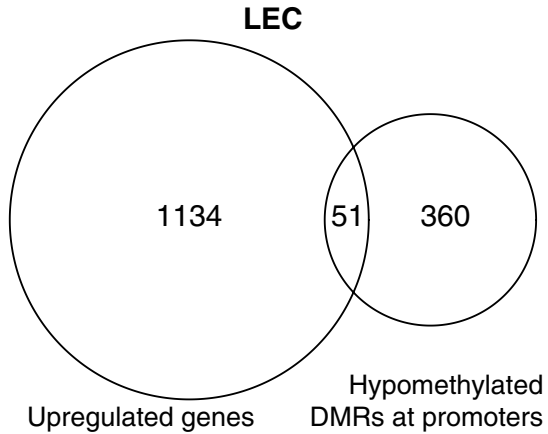

f

CD34

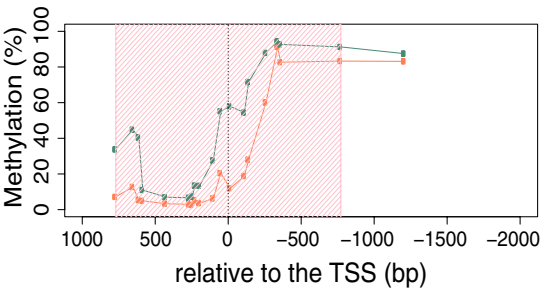

g

PROX1

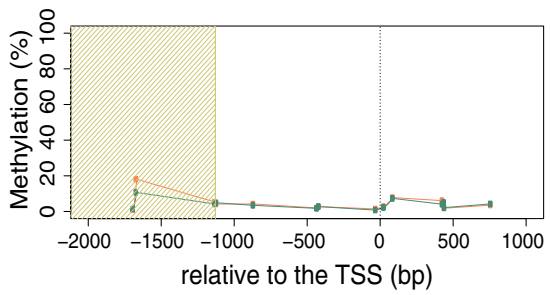

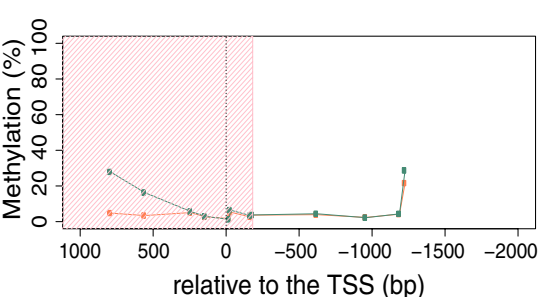

PDPN

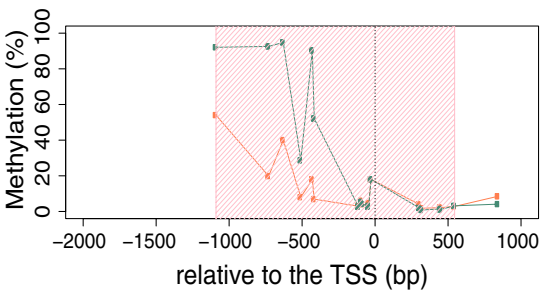

FLT1

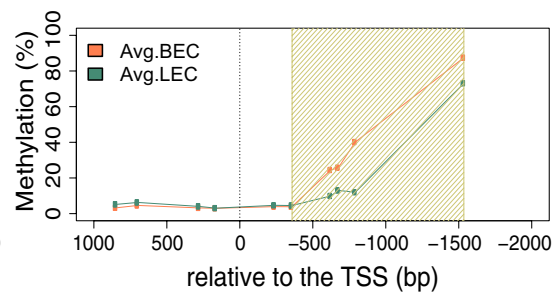

FLT4

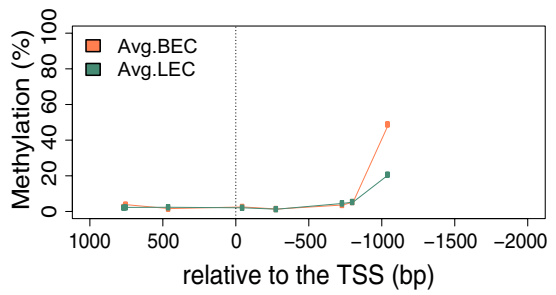


4Fig. 3 Elevated expression of endothelial lineage markers is not strictly controlled by DNA methylation of promoter regions. a Volcano plot representing the hypomethylated $\mathrm{CpG}$ loci in BECs (orange) and LECs (green), with a cutoff of $\log 2$ fold-change $(\mathrm{FC})<1$ and adjusted $p$ values $<0.05$. $\mathbf{b}$ and $\mathbf{c}$ Violin plots showing the methylation levels at the indicated genic regions (TSS1500, TSS200, 5'UTR, 1st exon, gene body, exon boundaries and 3'UTR) of blood vascular- (b) and lymphatic-specific (c) genes in BECs (orange) and LECs (green). The numbers in bracket indicate the numbers of $\mathrm{CpG}$ loci included in each genic location. Venn diagrams showing the genes with upregulated expression and hypomethylated DMRs at their promoters in BECs (d) and LECs (e). $\mathbf{f}$ and $\mathbf{g}$ Detailed view of the methylation patterns at the promoters $(-2 \mathrm{~kb}$ to $+1 \mathrm{~kb}$ relative to the TSS at position 0) of the selected markers. The methylation levels at the interrogated $\mathrm{CpG}$ loci in BECs and LECs are denoted by connected dots in orange and green, respectively. The shaded regions highlight the DMRs which were hypomethylated in BECs (orange) and LECs (green)

the respective cell type. Unexpectedly, the FLT1 promoter was hypermethylated in BECs compared to LECs. Likewise, the PDPN promoter was more heavily methylated in LECs whereas the promoter region of FLT4 displayed comparable methylation patterns in BECs and LECs (Fig. 3f, g).

\section{BCL6 and MEF2C as key transcription regulators potentially governing endothelial cell identity}

Enhancers serve as the docking sites for transcription factor binding, and cell type-specific enhancers play an indispensable role in lineage determination [30]. In particular, DNA methylation levels of intronic enhancers have been shown to inversely correlate with gene expression across tissues and species [31]. Based on the differential methylation analysis, we identified more than 600 DMRs located in enhancer regions, which might represent potential regulatory elements involved in the patterning of lineage-specific transcriptomes. Of note, we found hypomethylated DMRs at the intronic enhancer regions of a handful of markers differentially expressed in BECs versus LECs (Fig. S3e, f, Supplementary Table 2). To gain an integrative view of epigenetic alterations and the differential transcriptomic profiles required for maintenance of lineage-specific EC identity, we next performed transcription factor binding motif analyses. We first selected the hypomethylated enhancer DMRs lacking H3K27me3 coverage (Supplementary Table 3), which were then used in parallel with the upregulated genes as inputs to search for enriched motifs (summarized in Supplementary Table 4). Subsequently, we assessed the histone states and the expression levels of the transcription factors identified by both motif analyses (Fig. 4a). We identified the transcription repressor BCL6 and the transcription enhancer factor MEF2C as potential key upstream regulators in BECs and LECs, respectively (Fig. 4b). Both transcription factors showed 'active' histone states (Fig. 4b) with increased RNA expression (Fig. 4c, d, Fig. S4a, b) and protein levels
(Fig. 4e, Fig. S4c, d) in the respective cell type, suggesting their contribution to the transcriptomic and epigenetic regulation of endothelial lineage specificity. Most intriguingly, we observed differential expression patterns of certain reported interacting partners of BCL6 and MEF2C, including class II histone deacetylases (HDAC4 and HDAC9), SOX18 and KLF4, which were upregulated in the respective cell type (Fig. S4e) [32-35].

\section{Treatment with epigenetic drugs selectively promotes expression of blood vessel markers in lymphatic endothelial cells}

We focused our attention on the epigenetic profiles of 3 BEC and 3 LECs markers, all of which showed an 'active' histone state in the respective cell type. Notably, these lineage-specific markers adopted a more favorable histone composition and/or a hypomethylated promoter compared to the opposing cell type (Fig. 5a). To further investigate the relevance of these epigenetic modifications for endothelial lineage specificity, we treated BECs and LECs with epigenetic drugs. More in detail, cells were treated with inhibitors targeting EZH2 (GSK126), a histone methyltransferase responsible for the formation of the repressive $\mathrm{H} 2 \mathrm{~K} 27 \mathrm{me} 3$ histone mark (Fig. S5a), or DNA methyltransferases (5-AZA) that catalyze DNA methylation (Fig. S5b) [36]. We then studied the expression levels of CD34, ESAM and FLT1, as well as the lymphatic markers PROX1, PDPN and FLT4. The inhibition of H3K27 trimethylation by GSK126 strongly increased the expression levels of the blood vascular markers CD34, ESAM and FLT1 in LECs and to a lesser degree also in BECs (Fig. 5b). Likewise, DNA demethylation by 5-AZA treatment was also able to induce BEC marker expression in both cell types (Fig. 5b). In LECs, the expression of PROX1 and FLT4 was strongly increased by GSK126 treatment (Fig. 5c). On the other hand, 5-AZA treatment further enhanced the expression of PDPN and FLT4 in LECs (Fig. 5c). It is of interest that neither the blockade of H3K27 trimethylation nor inhibition of DNA methylation was able to upregulate the lymphatic markers PROX1 and PDPN in BECs, whereas we only observed a slight but significant increase in FLT4 expression (Fig. 5c).

Taken together with the histone enrichment and DNA methylation analyses, our findings suggest that LECs possess a more plastic phenotype compared to BECs. While blood vascular lineage markers were switched off during lymphatic specification, possibly via several epigenetic mechanisms, they could be reactivated in LECs by removing the repressive $\mathrm{H} 3 \mathrm{~K} 27$ trimethylation or DNA methylation. In contrast, BECs were more resistant to express lymphatic lineage markers, with the exception of FLT4 which was once expressed by the blood vasculature during embryonic development. 
a

Motif analysis of

DE gene list

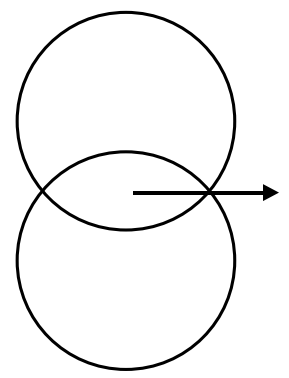

'Active' histone state $+$ Upregulated expression in the respective cell type

Motif analysis of hypomethylated enhancer

C

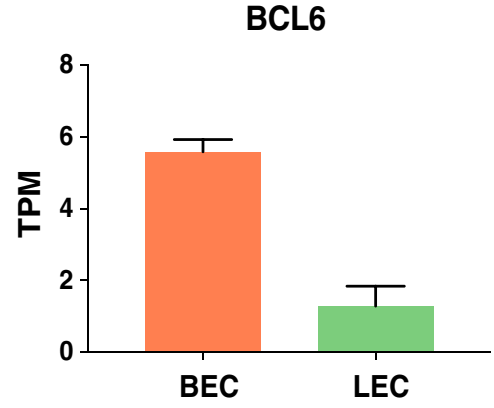

d

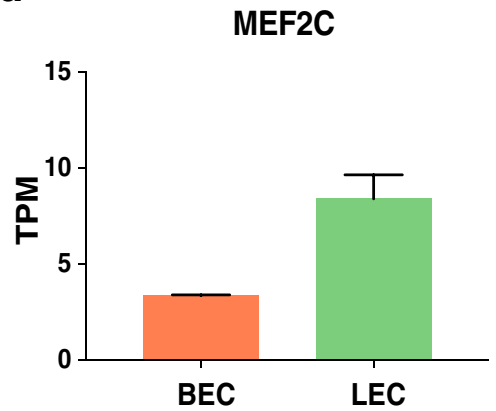

C

e b
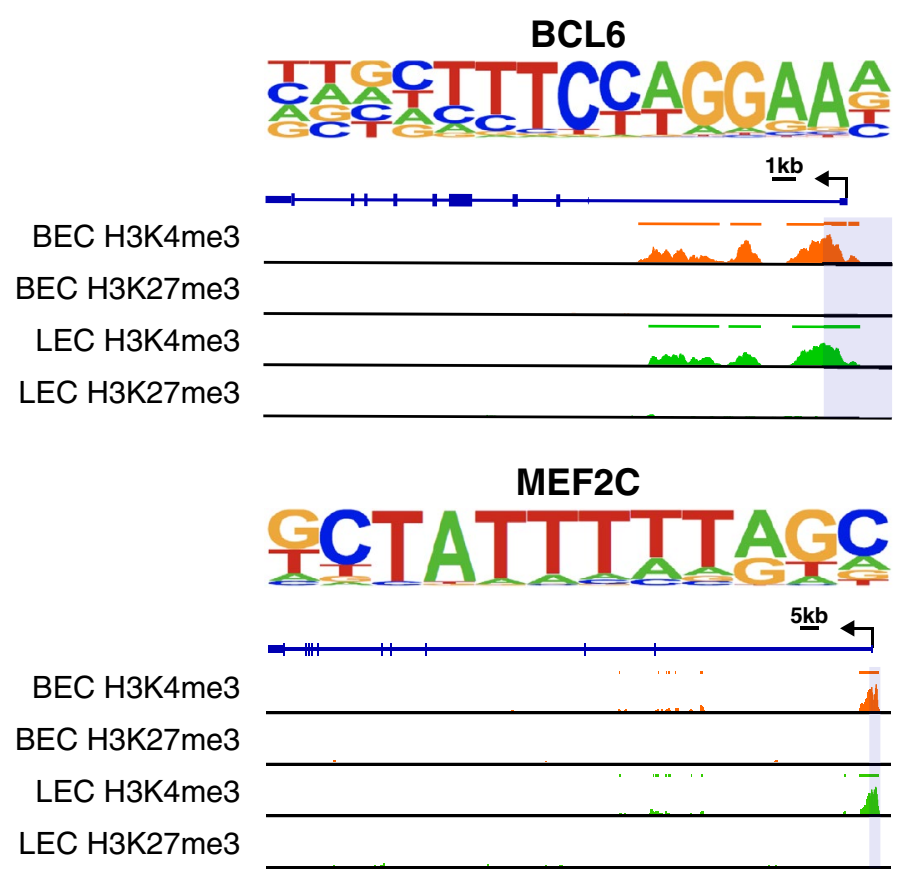
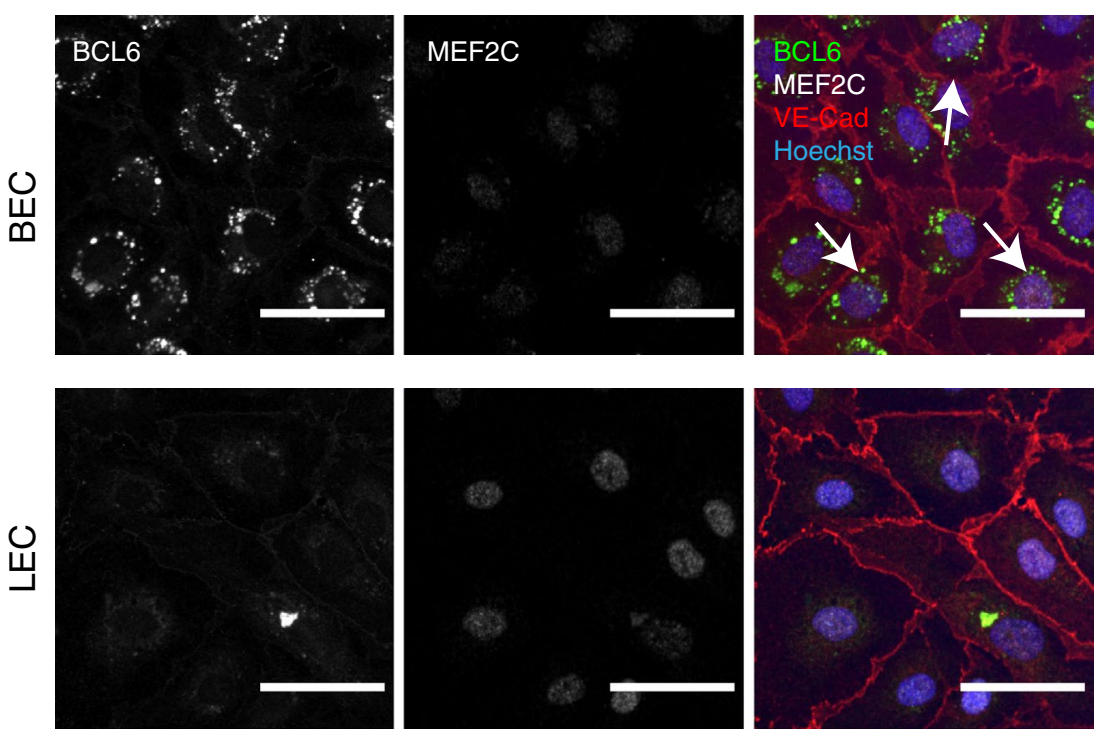

Fig. 4 Motif analysis reveals two potential transcription factors important for endothelial cell lineage identity. a Workflow of motif analyses and the transcription factor selection criteria. $\mathbf{b}$ The enriched binding motifs of BCL6 and MEF2C, as well as their histone composition in BECs and LECs, respectively. Colored bars at the top of the signal

\section{Discussion}

The lymphatic and blood vascular systems play fundamental roles in maintaining circulatory homeostasis, and tracks indicate the called ChIP-Seq peaks. Differential expression pattern of the transcription factors, BCL6 (c) and MEF2C (d), in BECs and LECs. e Representative immunofluorescence microscopy images of BCL6 and MEF2C in BECs and LECs ( $n=2 /$ cell line/donor). White arrows indicate BCL6-positive nuclei. Scale bar: $50 \mu \mathrm{m}$

impairment of their functions is associated with a large number of diseases, including cancer and chronic inflammatory diseases [37-39]. ECs possess unique transcriptional profiles that determine their lineage-specific functions. Considering 
a

\begin{tabular}{|c|c|c|c|ccc|}
\cline { 2 - 7 } \multicolumn{1}{c|}{} & \multicolumn{3}{c|}{ BECs } & \multicolumn{3}{c|}{ LECs } \\
\hline Markers & Histone state & Histone switch & $\begin{array}{c}\text { Hypomethylated } \\
\text { Promoter }\end{array}$ & Histone state & Histone switch & $\begin{array}{c}\text { Hypomethylated } \\
\text { Promoter }\end{array}$ \\
\hline CD34 & Active & Same & $\sqrt{ }$ & Active & Same & X \\
ESAM & Active & Gain & $\sqrt{ }$ & Active & Loss & X \\
\hline FLT1 & Active & Gain & $X$ & Repressed & Loss & $\sqrt{ }$ \\
\hline PROX1 & Repressed & Loss & $X$ & Active & Gain & $\sqrt{ }$ \\
PDPN & Unmarked & Loss & $\sqrt{ }$ & Active & Gain & X \\
FLT4 & Repressed & Loss & - & Active & Gain & - \\
\hline
\end{tabular}

b

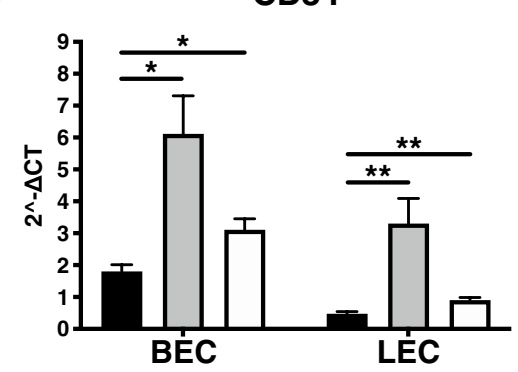

ESAM

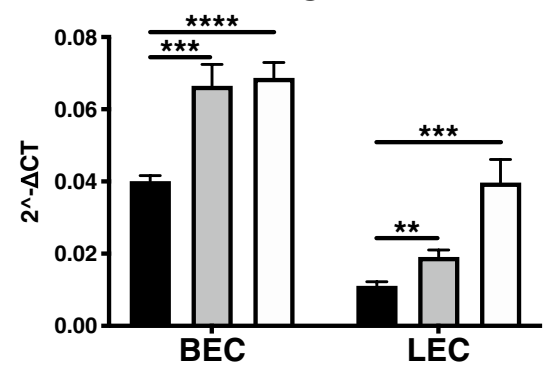

PDPN

C

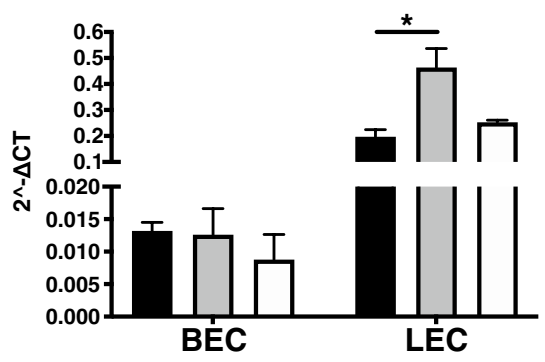

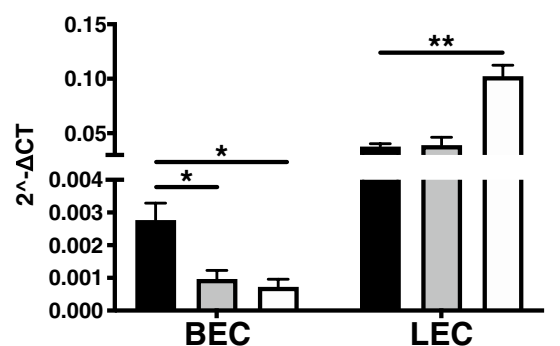

BEC

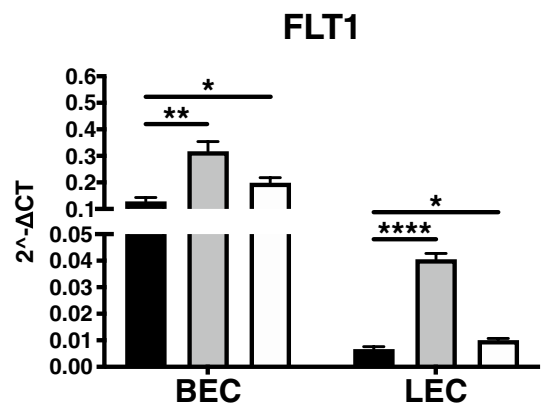

FLT1

CTRL $\square$ GSK126 $\square$ 5-AZA

FLT4

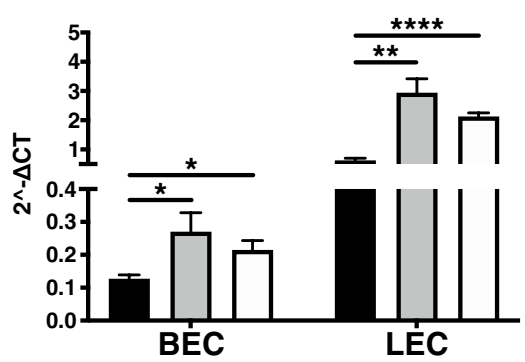

Fig. 5 Induction of blood vascular endothelial cell markers by epigenetic inhibitor treatment of lymphatic endothelial cells. a Summary table of the epigenetic landscape of the endothelial lineage-specific markers. After treatment for 7 consecutive days with GSK126, 5-AZA or DMSO (CTRL), BEC (b) and LEC (c) marker genes were

the increasingly recognized importance of epigenetic alterations in cellular differentiation processes, the epigenome may also play an important role in preserving the identity of terminally differentiated ECs. Our study provides the first comparative epigenome-wide profiling of human primary ECs and reveals distinctive epigenetic signatures of BECs and LECs. quantified by qPCR and shown as relative expression level using the $2^{-\triangle \mathrm{CT}}$ method. Data represent mean $+\mathrm{SD}$. Significance was determined by unpaired $t$ test $(n \geq 3$ replicate/group, representative of 3 independent experiments), $* p<0.05 ; * * p<0.01 ; * * * p<0.001$; $* * * * p<0.0001$

The distribution of methylation across the genome may vastly impact transcriptional activity, where hypermethylation of promoter regions is frequently associated with gene silencing [40]. Previously, a comparative DNA methylation analysis of BECs and LECs reported over 30,000 differentially methylated $\mathrm{CpG}$ sites, among which about $5 \%$ coincided with differentially expressed genes [20]. Similarly, we 
identified roughly $30,000 \mathrm{CpG}$ loci that were differentially methylated between the two EC types. Despite multiple differences in the experimental setups, two-thirds of the differentially expressed genes in BECs and LECs identified by Bronneke et al. could also be confirmed in the present study (data not shown) [20]. Similarly, more than half to two-thirds of differentially methylated probes identified by our analysis were congruently found in the above-mentioned study (data not shown) [20], indicating an overall consensus between both studies regarding gene expression and DNA methylation of cultured human dermal ECs.

When we evaluated the DMRs present in the promoters or intronic enhancers, there was only a small number of genes that adopted both increased gene expression and hypomethylated promoters or intronic enhancers. Nevertheless, the bisulfite conversion of DNA employed in our study does not discriminate between cytosine methylation and hydroxymethylation. DNA hydroxymethylation is tightly linked to gene expression [41]. One of the dioxygenases that catalyzes the conversion of methylated cytosines into hydroxymethylcytosine $(5 \mathrm{hmC})$ is the Tet Methylcytosine Dioxygenase 1 (TET1). Interestingly, TET1 was differentially expressed between LECs and BECs (Fig. 1d). It is thus of great interest to investigate in further studies the important differences in DNA hydroxymethylation between the two cell types. On the other hand, by exploiting ChIP-Seq profiling of H3K4me3 and H3K27me3 histone marks, we found that over one third of BEC-specific genes showed a more 'repressed' histone state in LECs. In contrast, only $9.2 \%$ of genes upregulated in LECs could be ascribed to a 'gain' of histone states, with $10.2 \%$ even labeled with a 'loss' of favorable histone compositions. This suggests that the suppression of genes related to BEC identity, via the tuning of histone modifications, is crucial to the maintenance of the lymphatic lineage.

The induction of PROX1 expression during early embryonic development is considered to be the key driver of lymphatic fate determination [3, 42]. It has previously been reported that PROX1 acts as a binary switch by suppressing BEC lineage-specific genes [19]. Conversely, LECs could be partially reprogrammed into BECs when this brake was released [43]. Our study reveals that PROX1 expression is tightly regulated at the level of epigenetic modifications, by displaying a more 'active' histone state and a hypomethylated promoter in LECs. The finding that epigenetic drug treatment did not induce the expression of PROX1 in BECs reflects the importance of the lineage-specific expression of this master regulator of lymphatic specification. Similarly, PDPN expression could not be induced by 5-AZA and GSK126 treatment of BECs, in agreement with the major biological function of PDPN in promoting platelet aggregation, coagulation and thrombus formation when in contact with blood [44, 45]. FLT4 expression has been previously reported to be upregulated in blood vessels of tumors and healing wounds [11, 12, 46, 47]. Importantly, the level of FLT4 was elevated in BECs after the inhibition of DNA or histone methyltransferase, which may be in agreement with the developmental derivation of LECs from BECs, also indicating potential mechanisms underlying the aberrant expression of FLT4 in pathological conditions. In spite of the differential DNA methylation and histone composition profiles, all three BEC markers were upregulated in LECs by 5-AZA and GSK126 treatments, indicating the plasticity of LECs in adopting a blood vessel-like phenotype via epigenetic remodeling.

Besides the broad coverage over promoter regions, the Infinium MethylationEPIC array also features an additional coverage of intergenic and intragenic enhancer $\mathrm{CpG}$ loci. Tissue-specific DNA hypomethylation at enhancer regions is crucial for enhancer activity and strongly associates with marker expression [29, 48-50]. By conducting a metaanalysis of the three profiling techniques performed in this study, searching for potential upstream regulators using the hypomethylated enhancer regions together with the differential gene expression patterns and histone composition, we identified two transcription factors that potentially govern EC identity: the transcriptional repressor BCL6 (B-cell lymphoma 6) in BECs and the transcription enhancer factor MEF2C in LECs. BCL6 has previously been reported to be expressed by ECs [51] and to suppress angiogenic sprouting via the NOTCH signaling pathway [52]. Nevertheless, its role in the maintenance of the blood vascular endothelial lineage has not been described thus far. Notably, several of its interaction partners belong to the family of histone deacetylases (HDACs) that are essential for chromatin remodeling [34]. Thus, it would be of interest to dissect the participation of this transcriptional complex in monitoring the BEC epigenome in future studies. The lymphatic transcription regulator MEF2C belongs to the myocyte enhancer factor-2 (MEF2) family of MADS-box transcription factors that is often expressed in skeletal muscle cells [53]. Of note, MEF2C expression is indispensable for embryonic vascular development since its targeted deletion results in severe vascular defects and even lethality in mice [54]. The SOX18/KLF4-PROX1 axis is fundamental for VEGF-C/ VEGFR-3 (FLT4) signaling and for the transdifferentiation of lymphatic progenitor cells from the cardinal veins during embryonic development $[55,56]$. As studies have shown that MEF2C directly influences the transcriptional level of KLF4 [57] and the DNA-binding activity of SOX18 [58], it is plausible to speculate that MEF2C might also be involved in maintaining the lineage specificity of LECs and in shaping the lymphatic epigenome, potentially via interaction with HDACs [59].

The use of cultured cells in the current study may represent a limitation when performing transcriptomic and epigenetics analyses. In fact, previous studies have reported that 
cultured human and mouse ECs alter their gene expression profiles as compared to their native state in situ [60-62], along with major changes in epigenetic modifications upon culturing conditions $[62,63]$. Nevertheless, our study, in agreement with those by Wick et al. and Amatschek et al. $[60,61]$, revealed that several of the major lineage-specific endothelial markers maintained their expression profiles in vitro, and were finely regulated by DNA methylation and histone modifications. These findings indicate that such an epigenetic memory is crucial for the maintenance of endothelial lineage specificity and function. In addition, LECs possess a more plastic phenotype compared to BECs, as highlighted by the resistance of BECs to upregulate the two key lymphatic-specific functional genes PROX1 and PDPN after epigenetic drug treatments. It will be of great interest to investigate epigenetic modifications of these distinct vascular beds in vivo under chronic pathological conditions such as inflammatory diseases and cancer.

\section{Materials and methods}

\section{Cell culture}

Cells were isolated as previously described [18]. Briefly, human neonatal foreskins were obtained after routine circumcisions. After enzymatic digestion, the epidermis was removed and dermal cells were mechanically released. CD34+ BECs were isolated and purified using an antihuman CD34 antibody (BD Pharmingen) conjugated to immunomagnetic beads (Dynal, Invitrogen). Thereafter, the remaining CD34- cells were incubated with immunomagnetic beads-conjugated anti-human CD31 antibody (Dynal, Invitrogen) to select for LECs. Cells were cultured under standard culture conditions $\left(37{ }^{\circ} \mathrm{C}\right.$ and $5 \% \mathrm{CO}_{2}$ ) on collagen type-I (Advanced BioMatrix)-coated dishes $(50 \mu \mathrm{g} /$ $\mathrm{mL}$ ) in EBM medium (Lonza) containing 20\% FBS (Gibco), $1 \%$ penicillin/streptomycin (Gibco), $2 \mathrm{mmol} / \mathrm{L}$ L-glutamine (Gibco) and $10 \mu \mathrm{g} / \mathrm{mL}$ hydrocortisone (Sigma-Aldrich). LEC culture medium was supplemented with $25 \mu \mathrm{mol} / \mathrm{mL}$ cAMP (Sigma-Aldrich), while for BEC culture medium, endothelial cell growth supplement (PromoCell) was added, as previously described [18]. Cells were passaged every 5 days, sub-confluent cells between passage 6 and 7 were used for all experiments. All cells were routinely tested for mycoplasma contamination using the MycoScope PCR Mycoplasma Detection Kit (Genlantis).

\section{Flow cytometry}

Endothelial cells were detached, washed with FACS buffer (DPBS with 2\% FBS and $1 \mathrm{mM}$ EDTA), and stained with Alexa647-conjugated mouse anti-human podoplanin (1:70, clone 18H5, NB600-1013AF647, Novus Biologicals) and PE-conjugated mouse anti-human CD31 antibodies (1:20, clone WM59, BD Pharmingen) in FACS buffer for $30 \mathrm{~min}$ at $4{ }^{\circ} \mathrm{C}$. After a wash with FACS buffer, endothelial cells were acquired on a Cytoflex S (Beckman Coulter). Analysis was performed using the FlowJo software v10.5.3 (BD Biosciences).

\section{RNA sequencing}

RNA was isolated from donor-matched BECs and LECs at passage 6 using the NucleoSpin RNA kit (Macherey-Nagel) according to the manufacturer's instructions. From $100 \mathrm{ng}$ of high-quality RNA, cDNA libraries were prepared and subjected to paired-end (100 bp) sequencing for 50 million reads per sample on average. Reads were aligned to the iGenomes UCSC hg 19 build using STAR v2.4.2a [64] and blacklisted regions were removed by BEDTools v2.25.0 [65]. Mapped reads were assigned to expression counts using the iGenomes annotation file and featureCounts from the Rsubread package (v1.26.1) [66]. All meta-analyses were centered on the genomic coordinates of expressed genes (Transcripts Per Million; TPM $>0$ ). Differentially expressed genes were defined by DESeq2 (v1.16.1) [67] with the cutoff of false discovery rate (FDR) $<0.01$ and $\log 2$ fold-change $(\log 2 \mathrm{FC})>1$. Pearson correlation coefficients between biological replicates were computed using normalized counts. A heatmap of differentially expressed epigenetic modifiers was generated with ggplot2 (v3.2.0).

\section{RNA isolation, reverse transcription, and qPCR}

Cultured LECs and BECs were lysed and RNA was extracted using the Nucleospin RNA kit (Macherey-Nagel) according to manufacturer's instructions. RNA concentrations were measured using a NanoDrop ND-1000 spectrophotometer (Witec) and retrotranscribed using the High-Capacity cDNA Reverse Transcription Kit (Applied Biosystems). Gene expression in BECs and LECs was measured by qPCR using the PowerUp SYBR green master mix (Thermo Fisher) on a QuantStudio 7 Flex system. GAPDH served as an internal control. Relative expression of genes was calculated according to the $2^{-\triangle C T}$ formula. Primer sequences were: CD34fwd: TCC CAA AAG ACC CTG ATT GC; CD34-rev: AAT AGC CAG TGA TGC CCA AGA; ESAM-fwd: CAC CAG CAT TAG ATG TCA TCC ESAM-rev: CCT TGC AGA CAT AGA CTC CA; FLT1-fwd: CCC TTA TGA TGC CAG CAA GTG; FLT1-rev: CCA AAA GCC CCT CTT CCA A; PROX1-fwd: ACA AAA ATG GTG GCA CGG A; PROX1rev: CCT GAT GTA CTT CGG AGC CTG; PDPN-fwd: CAG TTG AGA AAG ATG GTT TGT C; PDPN-rev: GAT GAT TGC ACC AAT GAA GC; FLT4-fwd: TCT GCT ACA GCT TCC AGG TGG; FLT4-rev: GCA GCC AGG TCT 
CTG TGG AT; MEF2C-fwd: GAA CGT AAC AGA CAG GTG AC; MEF2C-rev: CGC AAT CTC ACA GTC ACA C; BCL6-fwd: GAT GAG ATT GCC CTG CAT TT; BCL6-rev: TTC TTC CAG TTG CAG GCT TT; GAPDH-fwd: GAA ATC CCA TCA CCA TCT TCC AGG; GAPDH-rev: GAG CCC CAG CCT TCT CCA TG.

\section{ChIP-sequencing}

BECs and LECs were fixed for $8 \mathrm{~min}$ at room temperature using $1 \%$ of formaldehyde. The ChIP-Seq experiment was conducted by the Diagenode ChIP-Seq Profiling service (G02010000, Diagenode). Chromatin was prepared using the iDeal ChIP-Seq kit for histones (C01010059, Diagenode). Chromatin was sheared using a Bioruptor Pico sonication device (B01060001, Diagenode) combined with a Bioruptor Water cooler for 12 cycles using 30" [ON] 30" [OFF] settings. Shearing was performed in $0.65 \mathrm{~mL}$ Bioruptor Pico Microtubes (C30010011, Diagenode) with 1 million cells in $100 \mu \mathrm{L} .50 \mu \mathrm{L}$ of this chromatin was used to assess the size of the DNA fragments obtained by High Sensitivity NGS Fragment Analysis Kit (DNF-474) on a Fragment Analyzer (Advanced Analytical Technologies, Inc.).

ChIP was performed using an IP-Star®Compact Automated System (B03000002, Diagenode) following the protocol of the aforementioned kit. Chromatin corresponding to 1 million cells was immunoprecipitated using the following antibodies: H3K27me3 (C15410195, Diagenode) and H3K4me3 (C15410003, Diagenode). Chromatin corresponding to $1 \%$ was set apart as input. The DNA after reverse cross-linking was quantified using Qubit dsDNA HS Assay Kit (Q32854, Thermo Fisher Scientific). qPCR analyses were performed to check ChIP efficiency using KAPA SYBR FAST (Sigma-Aldrich) on a LightCycler 96 System (Roche). Libraries were prepared from input and immunoprecipitated DNA using the MicroPlex Library Preparation Kit v2 (12 indices) (C05010013, Diagenode). Library amplification was assessed using the High Sensitivity NGS Fragment Analysis Kit (DNF-474) on a Fragment Analyzer. Libraries were then purified using Agencourt AMPure XP (Beckman Coulter) and quantified using the Qubit dsDNA HS Assay Kit. Finally, their fragment size was analyzed by High Sensitivity DNA Analysis Kits on a 2100 Bioanalyzer system (Agilent). When the proportion of fragments $>1 \mathrm{~Kb}$ was too high, libraries were subjected to a double size selection with Agencourt AMPure XP and newly quantified and analyzed to assess their final size.

The libraries were subjected to single-read sequencing with at least 15 million reads per sample for input, 8 million reads for the $\mathrm{H} 3 \mathrm{~K} 4 \mathrm{me} 3$ mark and 35 million reads for H3K27me3 modification. Sequencing reads were aligned to the hg 19 genome using bowtie2 v2.2.3 [68], deduplicated with Picard v1.139 and filtered for blacklisted regions. The correlation coefficients between biological replicates of each histone mark were computed using deepTools v3.3.0 'multiBamSummary' and heatmaps were generated with 'plotCorrelation' [69]. MACS2 v2.1.1 was used for calling broad H3K27me3 peaks (-broad -m 350 -broad-cutoff 0.01 -nomodel -extsize 260) and narrow $\mathrm{H} 3 \mathrm{~K} 4 \mathrm{me} 3$ peaks (-m 750 -q 0.01 -nomodel -extsize 220), normalized signal was quantified as fragment per million reads [70]. Fold enrichment (bdgcmp-m FE) comparing histone occupancy against input controls was calculated. The independent signal tracks of biological replicates were combined using the unionbedg sub-command in BEDTools to generate an average track for visualization in the Integrative Genomics Viewer (IGV; v2.4.14) [71]. Peaks identified in both biological replicates were retained for downstream analyses. The distribution of histone enrichment peaks in either cell type across different genomic regions was assessed by the ChIPseeker package (v1.21.0) [72]. Enriched peaks were classified into promoter-overlapping $(-2 \mathrm{~kb}$ to $+1 \mathrm{~kb}$ relative to the TSS) or gene body-spanning regions, which were subsequently utilized for histone state grading of individual genes.

The DiffBind package (v2.13.0) was used to perform differential peak analysis $[73,74]$ with both DEseq2 and EdgeR $[75,76]$ methods. We filtered for differentially enriched peaks with a cutoff of fold enrichment $>1$ and FDR $<0.05$ in both methods.

\section{DNA methylation}

DNA from BECs and LECs was extracted using the DNeasy Blood \& Tissue Kit (Qiagen). DNA was bisulfite-converted and hybridized onto the Infinium MethylationEPIC BeadChip array that interrogates the methylation levels of over $850,000 \mathrm{CpG}$ sites across the genome. With regard to the genomic context of the $\mathrm{CpG}$ sites, $34 \%$ of probes are located at the promoters, $39 \%$ of probes at intragenic $\mathrm{CpGs}$ and the remaining $26.9 \%$ of probes in the intergenic regions that include enhancers (each covered by $1-3 \mathrm{CpG}$ sites) defined by the ENCODE and the FANTOM5 projects [77]. The import of raw data, filtering of probes with detection $p$ values $(<0.01)$, background correction, Subset-quantile Within Array Normalization (SWAN) and genomic coordinate annotations (hg19) were performed with the minfi package (v1.28.3) [78]. Potentially cross-hybridizing probes $(\geq 47$ nucleotide off-target homology) and probes overlapping with known SNPs (minor allele frequency $\geq 5 \%$ ) defined by both $[79,80]$ were also removed, resulting in 811,245 probes remaining for downstream analysis. The $\beta$-values [methylated/(unmethylated + methylated)], representing the proportional methylated signals, were used for graphical visualization and the $M$-values $[M=\log 2(\beta / 1-\beta)]$ were used for testing statistical significance. Linear models were fitted for 
differential methylation analysis of individual probes $(\mathrm{CpG}$ sites) with the limma package (v3.38.3) [81], where Benjamini-Hochberg (BH) adjusted $p$ values below 0.05 were considered significant. DMRcate (v1.18.0) was utilized to identify differentially methylated regions (DMRs), constituted of minimum $3 \mathrm{CpG}$ sites with Stouffer transformed FDR below 0.05 [82]. DMRs were subsequently assigned to genes with an overlapping promoter region $(-2 \mathrm{~kb}$ to $+1 \mathrm{~kb}$ relative to the TSS). Enhancer DMRs were defined as regions comprised of or at least containing significantly differentially methylated probes targeting enhancers.

\section{Transcription factor binding motif analyses}

Transcription factor binding motif analysis of upregulated genes was performed with HOMER (v4.10) 'findMotifs.pl' (-start -2000 -end 1000) [83]. In parallel, we used HOMER 'findMotifsGenome.pl' (-size given) for motif analysis of hypomethylated enhancer DMRs devoid of the H3K27me3 mark. The transcription factors yielded from both motif analyses were then filtered for their histone states and gene expression patterns, where those with an 'active' histone state and upregulated expression in the respective cell type were identified as potential upstream transcriptional regulators.

\section{Immunofluorescence stainings}

Cells were seeded into wells of a 24-well plate containing glass slides and cultured until confluence. Cells were fixed with 4\% PFA for $10 \mathrm{~min}$ at room temperature, blocked in blocking solution (PBS, $0.05 \%$ Tween $20,0.1 \%$ Triton-X, $5 \%$ donkey serum, 5\% BSA) and stained with primary antibodies (in blocking solution) overnight at $4{ }^{\circ} \mathrm{C}$, followed by washing in PBS and incubation with secondary antibodies for $1 \mathrm{~h}$ at room temperature. After washing, glass slides were mounted using Mowiol (Sigma-Aldrich). Primary antibodies were: rabbit anti-MEF2C (1:400, Cell Signaling, 5030), mouse anti-BCL6 (1:100, Santa Cruz, sc-7388) and goat anti-VE-cadherin (1:100, R\&D, AF938). Secondary antibodies were: donkey anti-goat AlexaFluor594, donkey antimouse AlexaFluor488, donkey anti-rabbit AlexaFluor647 (1:1000, all from Life Technologies). Confocal images were taken with an LSM780 microscope (Zeiss).

\section{Western blot}

Passage and donor-matched LECs and BECs were lysed with lysis buffer (25 mM HEPES, $5 \mathrm{mM}$ EDTA, 1\% triton-X, $150 \mathrm{mM} \mathrm{NaCl}, 10 \%$ glycerol, complete protease inhibitor cocktail) and then centrifuged at 13,000 rpm for $20 \mathrm{~min}$ at $4^{\circ}$ to collect the supernatant. Protein concentration of lysates was determined using the Microplate BCA protein assay kit
(Thermo Fisher), according to the manufacturer's instruction. Proteins were separated by SDS-PAGE on $4-12 \%$ NuPAGE Bis-Tris protein gels (Invitrogen) and transferred to a PVDF membrane (Immobilon-P, Millipore). Membranes were blocked in $5 \%$ milk in TBS $+0.1 \%$ Tween 20 , and incubated with primary antibodies (rabbit anti-MEF2C 1:1000, Cell Signaling, 5030; mouse anti-BCL6, 1:500, eBioscience, GI191E; rabbit anti-b-Actin 1:5000, Abcam, ab8227; rabbit anti-H3K27me3 1:1000, Diagenode, C15410195, rabbit anti-DNMT 1:1000, Cell Signaling, 5032) in 5\% milk in TBS $+0.1 \%$ Tween 20 , followed by washes and incubation with secondary antibodies (goat anti-rabbit and goat antimouse both 1:5000, Dako, labeled with HRP). Signal was developed with ECL Prime (GE Healthcare) and imaged on a ChemiDoc imaging system (Bio-Rad).

\section{Perturbation of gene expression of lymphatic and blood vascular endothelial cells}

Cells were seeded and treated the following day with $0.2 \mu \mathrm{M}$ of GSK126 (xcessbio) or $5 \mu \mathrm{M}$ of 5-Aza-2'-deoxycytidine (5-AZA; Sigma-Aldrich) for 7 consecutive days. Media were changed every other day with fresh media supplemented with the corresponding treatment. At the end of the treatment, cells were lysed and RNA was extracted for qPCR quantification as described above.

\section{Statistical analyses}

Statistical analyses were performed using Prism v7.0a (GraphPad Software Inc.). Data are shown as mean +SD. To determine statistical significance, a 2-tailed, unpaired Student's $t$ test was performed. Differences were considered statistically significant at $p<0.05$. Bioinformatic analyses were performed with Python v2.7.6 and R v3.4.0.

Acknowledgements The authors thank Dr. Lothar Dieterich for helpful scientific discussions and Sandra Kryenbühl for excellent technical assistance. We thank the microarray unit of the DKFZ Genomics and Proteomics Core Facility for providing the Illumina Human Methylation arrays and related services. This study was supported by the Swiss National Science Foundation (Grant Nos. 310030_166490 and 310030B_185392) and the European Research Council (Advance Grant LYVICAM).

Funding Open access funding provided by Swiss Federal Institute of Technology Zurich.

Data availability RNA-Seq (E-MTAB-8950), ChIP-Seq (E-MTAB-8951) and DNA methylation (E-MTAB-8952) data are accessible in ArrayExpress. 


\section{Compliance with ethical standards}

Conflict of interest The authors declare that they have no conflict of interest.

Open Access This article is licensed under a Creative Commons Attribution 4.0 International License, which permits use, sharing, adaptation, distribution and reproduction in any medium or format, as long as you give appropriate credit to the original author(s) and the source, provide a link to the Creative Commons licence, and indicate if changes were made. The images or other third party material in this article are included in the article's Creative Commons licence, unless indicated otherwise in a credit line to the material. If material is not included in the article's Creative Commons licence and your intended use is not permitted by statutory regulation or exceeds the permitted use, you will need to obtain permission directly from the copyright holder. To view a copy of this licence, visit http://creativecommons.org/licenses/by/4.0/.

\section{References}

1. Bautch VL, Caron KM (2015) Blood and lymphatic vessel formation. Cold Spring Harb Perspect Biol 7(3):a008268. https:// doi.org/10.1101/cshperspect.a008268

2. Cueni LN, Detmar M (2006) New insights into the molecular control of the lymphatic vascular system and its role in disease. J Invest Dermatol 126(10):2167-2177. https://doi.org/10.1038/ sj.jid.5700464

3. Wigle JT, Harvey N, Detmar M, Lagutina I, Grosveld G, Gunn MD, Jackson DG, Oliver G (2002) An essential role for Prox 1 in the induction of the lymphatic endothelial cell phenotype. Embo J 21(7):1505-1513. https://doi.org/10.1093/emboj/21.7.1505

4. Welsh JD, Kahn ML, Sweet DT (2016) Lymphovenous hemostasis and the role of platelets in regulating lymphatic flow and lymphatic vessel maturation. Blood 128(9):1169-1173. https:// doi.org/10.1182/blood-2016-04-636415

5. Hong YK, Detmar M (2003) Prox1, master regulator of the lymphatic vasculature phenotype. Cell Tissue Res 314(1):85-92. https://doi.org/10.1007/s00441-003-0747-8

6. Hong YK, Harvey N, Noh YH, Schacht V, Hirakawa S, Detmar M, Oliver G (2002) Prox 1 is a master control gene in the program specifying lymphatic endothelial cell fate. Dev Dynam 225(3):351-357. https://doi.org/10.1002/dvdy.10163

7. Yang Y, Oliver G (2014) Development of the mammalian lymphatic vasculature. J Clin Invest 124(3):888-897. https://doi. org/10.1172/Jci71609

8. Jha SK, Rauniyar K, Jeltsch M (2018) Key molecules in lymphatic development, function, and identification. Ann Anat 219:25-34. https://doi.org/10.1016/j.aanat.2018.05.003

9. Dejana E, Hirschi KK, Simons M (2017) The molecular basis of endothelial cell plasticity. Nat Commun. https://doi. org/10.1038/ncomms 14361

10. Ma WS, Oliver G (2017) Lymphatic endothelial cell plasticity in development and disease. Physiology 32(6):444-452. https ://doi.org/10.1152/physiol.00015.2017

11. Tammela T, Zarkada G, Wallgard E, Murtomaki A, Suchting S, Wirzenius M, Waltari M, Hellstrom M, Schomber T, Peltonen R, Freitas C, Duarte A, Isoniemi H, Laakkonen P, Christofori G, Yla-Herttuala S, Shibuya M, Pytowski B, Eichmann A, Betsholtz C, Alitalo K (2008) Blocking VEGFR-3 suppresses angiogenic sprouting and vascular network formation. Nature 454(7204):656-U668. https://doi.org/10.1038/nature07083

12. Valtola R, Salven P, Heikkila P, Taipale J, Joensuu H, Rehn M, Pihlajaniemi T, Weich H, deWaal R, Alitalo K (1999) VEGFR-3 and its ligand VEGF-C are associated with angiogenesis in breast cancer. Am J Pathol 154(5):1381-1390. https://doi. org/10.1016/S0002-9440(10)65392-8

13. Ladstatter S, Tachibana K (2019) Genomic insights into chromatin reprogramming to totipotency in embryos. J Cell Biol 218(1):70-82. https://doi.org/10.1083/jcb.201807044

14. Stylianou E (2019) Epigenetics of chronic inflammatory diseases. J Inflamm Res 12:1-14. https://doi.org/10.2147/Jir.S1290 27

15. Poli V, Fagnocchi L, Zippo A (2018) Tumorigenic cell reprogramming and cancer plasticity: interplay between signaling, microenvironment, and epigenetics. Stem Cells Int. https://doi. org/10.1155/2018/4598195

16. Schlereth K, Weichenhan D, Bauer T, Heumann T, Giannakouri E, Lipka D, Jaeger S, Schlesner M, Aloy P, Eils R, Plass C, Augustin HG (2018) The transcriptomic and epigenetic map of vascular quiescence in the continuous lung endothelium. Elife. https://doi.org/10.7554/eLife.34423

17. Sabbagh MF, Heng JS, Luo C, Castanon RG, Nery JR, Rattner A, Goff LA, Ecker JR, Nathans J (2018) Transcriptional and epigenomic landscapes of CNS and non-CNS vascular endothelial cells. Elife. https://doi.org/10.7554/eLife.36187

18. Hirakawa S, Hong YK, Harvey N, Schacht V, Matsuda K, Libermann T, Detmar M (2003) Identification of vascular lineage-specific genes by transcriptional profiling of isolated blood vascular and lymphatic endothelial cells. Am J Pathol 162(2):575-586. https://doi.org/10.1016/S0002-9440(10)63851-5

19. Petrova TV, Makinen T, Makela TP, Saarela J, Virtanen I, Ferrell RE, Finegold DN, Kerjaschki D, Yla-Herttuala S, Alitalo K (2002) Lymphatic endothelial reprogramming of vascular endothelial cells by the Prox-1 homeobox transcription factor. Embo J 21(17):4593-4599. https://doi.org/10.1093/emboj/cdf470

20. Bronneke S, Bruckner B, Peters N, Bosch TCG, Stab F, Wenck H, Hagemann S, Winnefeld M (2012) DNA methylation regulates lineage-specifying genes in primary lymphatic and blood endothelial cells. Angiogenesis 15(2):317-329. https://doi.org/10.1007/ s10456-012-9264-2

21. Shirodkar AV, St Bernard R, Gavryushova A, Kop A, Knight BJ, Yan MS, Man HS, Sud M, Hebbel RP, Oettgen P, Aird WC, Marsden PA (2013) A mechanistic role for DNA methylation in endothelial cell (EC)-enriched gene expression: relationship with DNA replication timing. Blood 121(17):3531-3540. https://doi. org/10.1182/blood-2013-01-479170

22. Barrero MJ, Boue S, Belmonte JCI (2010) Epigenetic mechanisms that regulate cell identity. Cell Stem Cell 7(5):565-570. https:// doi.org/10.1016/j.stem.2010.10.009

23. Plass C, Pfister SM, Lindroth AM, Bogatyrova O, Claus R, Lichter $P$ (2013) Mutations in regulators of the epigenome and their connections to global chromatin patterns in cancer. Nat Rev Genet 14(11):765-780. https://doi.org/10.1038/nrg3554

24. Hyun K, Jeon J, Park K, Kim J (2017) Writing, erasing and reading histone lysine methylations. Exp Mol Med. https://doi. org/10.1038/emm.2017.11

25. Ruthenburg AJ, Allis CD, Wysocka J (2007) Methylation of lysine 4 on histone $\mathrm{H} 3$ : intricacy of writing and reading a single epigenetic mark. Mol Cell 25(1):15-30. https://doi.org/10.1016/j.molce 1.2006.12.014

26. Saksouk N, Simboeck E, Dejardin J (2015) Constitutive heterochromatin formation and transcription in mammals. Epigenetics Chromatin 8:3. https://doi.org/10.1186/1756-8935-8-3

27. Paksa A, Rajagopal J (2017) The epigenetic basis of cellular plasticity. Curr Opin Cell Biol 49:116-122. https://doi.org/10.1016/j. ceb.2018.01.003

28. Kim M, Costello J (2017) DNA methylation: an epigenetic mark of cellular memory. Exp Mol Med. https://doi.org/10.1038/ emm. 2017.10 
29. Jones PA (2012) Functions of DNA methylation: islands, start sites, gene bodies and beyond. Nat Rev Genet 13(7):484-492. https://doi.org/10.1038/nrg3230

30. Melamed P, Yosefzon Y, Rudnizky S, Pnueli L (2016) Transcriptional enhancers: transcription, function and flexibility. TranscrAustin 7(1):26-31. https://doi.org/10.1080/21541264.2015.11285 17

31. Anastasiadi D, Esteve-Codina A, Piferrer F (2018) Consistent inverse correlation between DNA methylation of the first intron and gene expression across tissues and species. Epigenet Chromatin. https://doi.org/10.1186/s13072-018-0205-1

32. Zhao X, Sternsdorf T, Bolger TA, Evans RM, Yao TP (2005) Regulation of MEF2 by histone deacetylase 4-and SIRT1 deacetylasemediated lysine modifications. Mol Cell Biol 25(19):8456-8464. https://doi.org/10.1128/Mcb.25.19.8456-8464.2005

33. Haberland M, Arnold MA, McAnally J, Phan D, Kim Y, Olson EN (2007) Regulation of HDAC9 gene expression by MEF2 establishes a negative-feedback loop in the transcriptional circuitry of muscle differentiation. Mol Cell Biol 27(2):518-525. https://doi. org/10.1128/Mcb.01415-06

34. Zhang H, Okada S, Hatano M, Okabe S, Tokuhisa T (2001) A new functional domain of Bcl6 family that recruits histone deacetylases. Bba-Mol Cell Res 1540(3):188-200. https://doi. org/10.1016/S0167-4889(01)00128-8

35. Lemercier C, Brocard MP, Puvion-Dutilleul F, Kao HY, Albagli O, Khochbin S (2002) Class II histone deacetylases are directly recruited by BCL6 transcriptional repressor. J Biol Chem 277(24):22045-22052. https://doi.org/10.1074/jbc.M201736200

36. McCabe MT, Ott HM, Ganji G, Korenchuk S, Thompson C, Van Aller GS, Liu Y, Graves AP, Della Pietra A, Diaz E, LaFrance LV, Mellinger M, Duquenne C, Tian XR, Kruger RG, McHugh CF, Brandt M, Miller WH, Dhanak D, Verma SK, Tummino PJ, Creasy CL (2012) EZH2 inhibition as a therapeutic strategy for lymphoma with EZH2-activating mutations. Nature 492(7427):108. https://doi.org/10.1038/nature11606

37. Zgraggen S, Ochsenbein AM, Detmar M (2013) An important role of blood and lymphatic vessels in inflammation and allergy. $\mathrm{J}$ Allergy (Cairo) 2013:672381. https://doi.org/10.1155/2013/67238 1

38. Ruddell A, Croft A, Kelly-Spratt K, Furuya M, Kemp CJ (2014) Tumors induce coordinate growth of artery, vein, and lymphatic vessel triads. BMC Cancer 14:354. https://doi. org/10.1186/1471-2407-14-354

39. Karaman S, Detmar M (2014) Mechanisms of lymphatic metastasis. J Clin Invest 124(3):922-928. https://doi.org/10.1172/JCI71 606

40. Kass SU, Landsberger N, Wolffe AP (1997) DNA methylation directs a time-dependent repression of transcription initiation. Curr Biol 7(3):157-165. https://doi.org/10.1016/s0960 -9822(97)70086-1

41. Gross JA, Pacis A, Chen GG, Drupals M, Lutz PE, Barreiro LB, Turecki G (2017) Gene-body 5-hydroxymethylation is associated with gene expression changes in the prefrontal cortex of depressed individuals. Transl Psychiatry. https://doi.org/10.1038/tp.2017.93

42. Wong BW, Zecchin A, Garcia-Caballero M, Carmeliet P (2018) Emerging concepts in organ-specific lymphatic vessels and metabolic regulation of lymphatic development. Dev Cell 45(3):289_ 301. https://doi.org/10.1016/j.devcel.2018.03.021

43. Johnson NC, Dillard ME, Baluk P, McDonald DM, Harvey NL, Frase SL, Oliver G (2008) Lymphatic endothelial cell identity is reversible and its maintenance requires Prox 1 activity. Gene Dev 22(23):3282-3291. https://doi.org/10.1101/gad.1727208

44. Cueni LN, Chen L, Zhang H, Marino D, Huggenberger R, Alitalo A, Bianchi R, Detmar M (2010) Podoplanin-Fc reduces lymphatic vessel formation in vitro and in vivo and causes disseminated intravascular coagulation when transgenically expressed in the skin. Blood 116(20):4376-4384. https://doi.org/10.1182/blood -2010-04-278564

45. Riedl J, Preusser M, Nazari PMS, Posch F, Panzer S, Marosi C, Birner P, Thaler J, Brostjan C, Lotsch D, Berger W, Hainfellner JA, Pabinger I, Ay C (2017) Podoplanin expression in primary brain tumors induces platelet aggregation and increases risk of venous thromboembolism. Blood 129(13):1831-1839. https://doi. org/10.1182/blood-2016-06720714

46. Paavonen K, Puolakkainen P, Jussila L, Jahkola T, Alitalo K (2000) Vascular endothelial growth factor receptor-3 in lymphangiogenesis in wound healing. Am J Pathol 156(5):1499-1504. https ://doi.org/10.1016/S0002-9440(10)65021-3

47. Witmer AN, van Blijswijk BC, Dai J, Hofman P, Partanen TA, Vrensen GFJM, Schlingemann RO (2001) VEGFR-3 in adult angiogenesis. J Pathol 195(4):490-497. https://doi.org/10.1002/ path. 969

48. Ehrlich KC, Paterson HL, Lacey M, Ehrlich M (2016) DNA hypomethylation in intragenic and intergenic enhancer chromatin of muscle-specific genes usually correlates with their expression. Yale J Biol Med 89(4):441-455

49. Fleischer T, Tekpli X, Mathelier A, Wang SX, Nebdal D, Dhakal HP, Sahlberg KK, Schlichting E, Borresen-Dale AL, Borgen E, Naume B, Eskeland R, Frigessi A, Tost J, Hurtado A, Kristensen VN, OS OBCRC (2017) DNA methylation at enhancers identifies distinct breast cancer lineages. Nat Commun. https://doi. org/10.1038/s41467-017-00510-x

50. Rinaldi L, Datta D, Serrat J, Morey L, Solanas G, Avgustinova A, Blanco E, Pons JI, Matallanas D, Von Kriegsheim A, Di Croce L, Benitah SA (2016) Dnmt3a and Dnmt3b associate with enhancers to regulate human epidermal stem cell homeostasis. Cell Stem Cell 19(4):491-501. https://doi.org/10.1016/j.stem.2016.06.020

51. Fan Y, Wang Y, Tang Z, Zhang H, Qin X, Zhu Y, Guan Y, Wang X, Staels B, Chien S, Wang N (2008) Suppression of pro-inflammatory adhesion molecules by PPAR-delta in human vascular endothelial cells. Arterioscler Thromb Vasc Biol 28(2):315-321. https://doi.org/10.1161/ATVBAHA.107.149815

52. Buchberger E, Payrhuber D, El Harchi M, Zagrapan B, Scheuba K, Zommer A, Bugyik E, Dome B, Kral JB, Schrottmaier WC, Schabbauer G, Petzelbauer P, Groger M, Bilban M, Brostjan C (2017) Inhibition of the transcriptional repressor complex Bcl-6/ BCoR induces endothelial sprouting but does not promote tumor growth. Oncotarget 8(1):552-564. https://doi.org/10.18632/oncot arget.13477

53. Wang DZ, Valdez MR, McAnally J, Richardson J, Olson EN (2001) The Mef2c gene is a direct transcriptional target of myogenic bHLH and MEF2 proteins during skeletal muscle development. Development 128(22):4623-4633

54. Lin Q, Lu JR, Yanagisawa H, Webb R, Lyons GE, Richardson JA, Olson EN (1998) Requirement of the MADS-box transcription factor MEF2C for vascular development. Development 125(22):4565-4574

55. Francois M, Caprini A, Hosking B, Orsenigo F, Wilhelm D, Browne C, Paavonen K, Karnezis T, Shayan R, Downes M, Davidson T, Tutt D, Cheah KSE, Stacker SA, Muscat GEO, Achen MG, Dejana E, Koopman P (2008) Sox 18 induces development of the lymphatic vasculature in mice. Nature 456(7222):643-669. https ://doi.org/10.1038/nature07391

56. Park DY, Lee J, Park I, Choi D, Lee S, Song S, Hwang Y, Hong KY, Nakaoka Y, Makinen T, Kim P, Alitalo K, Hong YK, Koh GY (2014) Lymphatic regulator PROX1 determines Schlemm's canal integrity and identity. J Clin Invest 124(9):3960-3974. https ://doi.org/10.1172/Jci75392

57. Maejima T, Inoue T, Kanki Y, Kohro T, Li G (2014) Direct evidence for pitavastatin induced chromatin structure change in the KLF4 gene in endothelial cells (vol 9, e96005, 2014). PLoS ONE. https://doi.org/10.1371/journal.pone.0099749 
58. Hosking BM, Wang SCM, Chen SL, Penning S, Koopman P, Muscat GEO (2001) SOX18 directly interacts with MEF2C in endothelial cells. Biochem Bioph Res Co 287(2):493-500. https ://doi.org/10.1006/bbrc.2001.5589

59. Messenguy F, Dubois E (2003) Role of MADS box proteins and their cofactors in combinatorial control of gene expression and cell development. Gene 316:1-21. https://doi.org/10.1016/S0378 $-1119(03) 00747-9$

60. Amatschek S, Kriehuber E, Bauer W, Reininger B, Meraner P, Wolpl A, Schweifer N, Haslinger C, Stingl G, Maurer D (2007) Blood and lymphatic endothelial cell-specific differentiation programs are stringently controlled by the tissue environment. Blood 109(11):4777-4785. https://doi.org/10.1182/blood-200610-053280

61. Wick N, Saharinen P, Saharinen J, Gurnhofer E, Steiner CW, Raab I, Stokic D, Giovanoli P, Buchsbaum S, Burchard A, Thurner S, Alitalo K, Kerjaschki D (2007) Transcriptomal comparison of human dermal lymphatic endothelial cells ex vivo and in vitro. Physiol Genomics 28(2):179-192. https://doi.org/10.1152/physi olgenomics.00037.2006

62. Sabbagh MF, Nathans J (2020) A genome-wide view of the dedifferentiation of central nervous system endothelial cells in culture. Elife. https://doi.org/10.7554/eLife.51276

63. Magnusson M, Larsson P, Lu EX, Bergh N, Caren H, Jern S (2016) Rapid and specific hypomethylation of enhancers in endothelial cells during adaptation to cell culturing. Epigenetics 11(8):614-624. https://doi.org/10.1080/15592294.2016.1192734

64. Dobin A, Davis CA, Schlesinger F, Drenkow J, Zaleski C, Jha S, Batut P, Chaisson M, Gingeras TR (2013) STAR: ultrafast universal RNA-seq aligner. Bioinformatics 29(1):15-21. https://doi. org/10.1093/bioinformatics/bts635

65. Quinlan AR, Hall IM (2010) BEDTools: a flexible suite of utilities for comparing genomic features. Bioinformatics 26(6):841-842. https://doi.org/10.1093/bioinformatics/btq033

66. Liao Y, Smyth GK, Shi W (2019) The R package Rsubread is easier, faster, cheaper and better for alignment and quantification of RNA sequencing reads. Nucleic Acids Res. https://doi. org/10.1093/nar/gkz114

67. Love MI, Huber W, Anders S (2014) Moderated estimation of fold change and dispersion for RNA-seq data with DESeq2. Genome Biol. https://doi.org/10.1186/s13059-014-0550-8

68. Langmead B, Salzberg SL (2012) Fast gapped-read alignment with Bowtie 2. Nat Methods 9(4):357-U354. https://doi. org/10.1038/Nmeth.1923

69. Ramirez F, Ryan DP, Gruning B, Bhardwaj V, Kilpert F, Richter AS, Heyne S, Dundar F, Manke T (2016) deepTools2: a next generation web server for deep-sequencing data analysis. Nucleic Acids Res 44(W1):W160-W165. https://doi.org/10.1093/nar/ gkw257

70. Zhang Y, Liu T, Meyer CA, Eeckhoute J, Johnson DS, Bernstein BE, Nussbaum C, Myers RM, Brown M, Li W, Liu XS (2008) Model-based analysis of ChIP-Seq (MACS). Genome Biol. https ://doi.org/10.1186/gb-2008-9-9-r137

71. Robinson JT, Thorvaldsdottir H, Wenger AM, Zehir A, Mesirov JP (2017) Variant review with the integrative genomics viewer. Cancer Res 77(21):E31-E34. https://doi.org/10.1158/0008-5472. Can-17-0337
72. Yu GC, Wang LG, He QY (2015) ChIPseeker: an R/Bioconductor package for ChIP peak annotation, comparison and visualization. Bioinformatics 31(14):2382-2383. https://doi.org/10.1093/bioin formatics/btv145

73. Stark R, Brown G (2011) DiffBind: differential binding analysis of ChIPSeq peak data. Bioconductor. https://doi.org/10.18129/ B9.bioc.DiffBind

74. Ross-Innes CS, Stark R, Teschendorff AE, Holmes KA, Ali HR, Dunning MJ, Brown GD, Gojis O, Ellis IO, Green AR, Ali S, Chin SF, Palmieri C, Caldas C, Carroll JS (2012) Differential oestrogen receptor binding is associated with clinical outcome in breast cancer. Nature 481(7381):389-393. https://doi.org/10.1038/ nature 10730

75. Robinson MD, McCarthy DJ, Smyth GK (2010) edgeR: a Bioconductor package for differential expression analysis of digital gene expression data. Bioinformatics 26(1):139-140. https://doi. org/10.1093/bioinformatics/btp616

76. McCarthy DJ, Chen Y, Smyth GK (2012) Differential expression analysis of multifactor RNA-Seq experiments with respect to biological variation. Nucleic Acids Res 40(10):4288-4297. https:// doi.org/10.1093/nar/gks042

77. Moran S, Arribas C, Esteller M (2016) Validation of a DNA methylation microarray for $850,000 \mathrm{CpG}$ sites of the human genome enriched in enhancer sequences. Epigenomics 8(3):389-399. https ://doi.org/10.2217/epi.15.114

78. Aryee MJ, Jaffe AE, Corrada-Bravo H, Ladd-Acosta C, Feinberg AP, Hansen KD, Irizarry RA (2014) Minfi: a flexible and comprehensive bioconductor package for the analysis of infinium DNA methylation microarrays. Bioinformatics 30(10):1363-1369. https ://doi.org/10.1093/bioinformatics/btu049

79. McCartney DL, Walker RM, Morris SW, McIntosh AM, Porteous DJ, Evans KL (2016) Identification of polymorphic and off-target probe binding sites on the Illumina Infinium MethylationEPIC BeadChip. Genom Data 9:22-24. https://doi.org/10.1016/j.gdata .2016.05.012

80. Pidsley R, Zotenko E, Peters TJ, Lawrence MG, Risbridger GP, Molloy P, Van Djik S, Muhlhausler B, Stirzaker C, Clark SJ (2016) Critical evaluation of the Illumina MethylationEPIC BeadChip microarray for whole-genome DNA methylation profiling. Genome Biol. https://doi.org/10.1186/s13059-016-1066-1

81. Ritchie ME, Phipson B, Wu D, Hu YF, Law CW, Shi W, Smyth GK (2015) limma powers differential expression analyses for RNA-sequencing and microarray studies. Nucleic Acids Res. https ://doi.org/10.1093/nar/gkv007

82. Peters TJ, Buckley MJ, Statham AL, Pidsley R, Samaras K, Lord RV, Clark SJ, Molloy PL (2015) De novo identification of differentially methylated regions in the human genome. Epigenet Chromatin. https://doi.org/10.1186/1756-8935-8-6

83. Heinz S, Benner C, Spann N, Bertolino E, Lin YC, Laslo P, Cheng JX, Murre C, Singh H, Glass CK (2010) Simple combinations of lineage-determining transcription factors prime cis-regulatory elements required for macrophage and B cell identities. Mol Cell 38(4):576-589. https://doi.org/10.1016/j.molcel.2010.05.004

Publisher's Note Springer Nature remains neutral with regard to jurisdictional claims in published maps and institutional affiliations. 